University of Tennessee Health Science Center

UTHSC Digital Commons

\title{
Effect of Sound Source Location and Spatial Hearing on the Vestibulo-Ocular Reflex (VOR)
}

\author{
Mary Easterday \\ University of Tennessee Health Science Center
}

Follow this and additional works at: https://dc.uthsc.edu/dissertations

Part of the Speech and Hearing Science Commons, and the Speech Pathology and Audiology Commons

\section{Recommended Citation}

Easterday, Mary (https://orcid.org/0000-0002-8744-475X), "Effect of Sound Source Location and Spatial Hearing on the Vestibulo-Ocular Reflex (VOR)" (2020). Theses and Dissertations (ETD). Paper 529. http://dx.doi.org/10.21007/etd.cghs.2020.0527. 


\title{
Effect of Sound Source Location and Spatial Hearing on the Vestibulo-Ocular Reflex (VOR)
}

\begin{abstract}
Accurate measurement of the vestibulo-ocular reflex (VOR) is imperative in differential diagnosis of vestibular disorders and balance function. However, the assessment protocol faces a number of limitations, including the need to control for extra- vestibular sensory factors such as hearing. Previous research has shown that the use of an auditory stimulus can have a significant effect on functional measures of balance, and many have contributed effects to be the result of spatial hearing. However, no studies have directly assessed the effect of speaker location on the VOR nor investigated correlations of functional spatial hearing with the VOR. Therefore, the aims of this study were to 1) assess the effect of speaker location on the VOR and 2) investigate if spatial hearing abilities are correlated with the strength of the VOR.

A between subjects repeated measures design was utilized with a child group (age 6-9 years) and an adult group (18-40 years). The rationale of the two groups was to compare differences in a group with underdeveloped localization abilities (child) and a group with matured localization abilities (adult). A total of 22 children and 23 adults participated in this study. Localization ability was measured using the root mean square (RMS) error method. VOR gain was measured using the rotational chair test at a rate of .08 $\mathrm{Hz}$ in the following auditory conditions: silent (insert earphones turned off), insert earphones turned on, external speaker at $0^{\circ}$ azimuth rotating with participant, and external stationary speaker. Order of testing was randomized to control for any order effects. An independent samples t-test confirmed a significant difference in RMS error between groups with the child group performing worse than the adults. Subsequent multivariate analysis of variance indicated a significant effect for speaker location with the external moving speaker having significantly lower gain and the external stationary speaker having significantly increased gain for both child and adult groups. Correlations were run for both groups for both fixed and moving speaker conditions. No correlation was seen in either condition for the child group, however, a positive correlation was seen for both conditions for the adult group, meaning as RMS error increased so did VOR gain.
\end{abstract}

These results indicate the possible need to control for environmental auditory stimulus location when undergoing vestibular assessment. However, further studies need to be performed to corroborate the evidence presented.

\section{Document Type}

Dissertation

Degree Name

Doctor of Philosophy (PhD)

\section{Program}

Speech and Hearing Science

Research Advisor

Patrick Plyler, PhD

\section{Keywords}

Assessment, Vestibular 


\section{Subject Categories}

Communication Sciences and Disorders | Medicine and Health Sciences | Speech and Hearing Science | Speech Pathology and Audiology 


\section{UNIVERSITY OF TENNESSEE HEALTH SCIENCE CENTER}

DOCTORAL DISSERTATION

\section{Effect of Sound Source Location and Spatial Hearing on the Vestibulo-Ocular Reflex (VOR)}

Author:

Mary Easterday
Advisor:

Patrick Plyler, Ph.D.

A Dissertation Presented for The Graduate Studies Council of

The University of Tennessee Health Science Center

in Partial Fulfillment of the Requirements for the Doctor of Philosophy degree from The University of Tennessee

in

Speech and Hearing Science: Audiology

College of Graduate Health Sciences

December 2020 
Copyright $@ 2020$ by Mary Easterday. All rights reserved. 


\section{DEDICATION}

This dissertation is dedicated to all those who supported and encouraged me through this arduous process. Most of all, this goes to my family at home and my family at the University of Tennessee. 


\section{ACKNOWLEDGEMENTS}

I would like to acknowledge Dr. Patrick Plyer for his invaluable mentorship and assistance; as well as committee members Drs. Ashley Harkrider, Patti Johnstone, James Lewis, Jefferey Fairbrother, and Devin McCaslin. I would also like to acknowledge Dr. Steven Doettl as the vestibular consultant and for his contribution to the research reported here. 


\begin{abstract}
Accurate measurement of the vestibulo-ocular reflex (VOR) is imperative in differential diagnosis of vestibular disorders and balance function. However, the assessment protocol faces a number of limitations, including the need to control for extravestibular sensory factors such as hearing. Previous research has shown that the use of an auditory stimulus can have a significant effect on functional measures of balance, and many have contributed effects to be the result of spatial hearing. However, no studies have directly assessed the effect of speaker location on the VOR nor investigated correlations of functional spatial hearing with the VOR. Therefore, the aims of this study were to 1) assess the effect of speaker location on the VOR and 2) investigate if spatial hearing abilities are correlated with the strength of the VOR.
\end{abstract}

A between subjects repeated measures design was utilized with a child group (age 6-9 years) and an adult group (18-40 years). The rationale of the two groups was to compare differences in a group with underdeveloped localization abilities (child) and a group with matured localization abilities (adult). A total of 22 children and 23 adults participated in this study. Localization ability was measured using the root mean square (RMS) error method. VOR gain was measured using the rotational chair test at a rate of $.08 \mathrm{~Hz}$ in the following auditory conditions: silent (insert earphones turned off), insert earphones turned on, external speaker at $0^{\circ}$ azimuth rotating with participant, and external stationary speaker. Order of testing was randomized to control for any order effects. An independent samples t-test confirmed a significant difference in RMS error between groups with the child group performing worse than the adults. Subsequent multivariate analysis of variance indicated a significant effect for speaker location with the external moving speaker having significantly lower gain and the external stationary speaker having significantly increased gain for both child and adult groups. Correlations were run for both groups for both fixed and moving speaker conditions. No correlation was seen in either condition for the child group, however, a positive correlation was seen for both conditions for the adult group, meaning as RMS error increased so did VOR gain.

These results indicate the possible need to control for environmental auditory stimulus location when undergoing vestibular assessment. However, further studies need to be performed to corroborate the evidence presented. 


\section{TABLE OF CONTENTS}

CHAPTER 1. INTRODUCTION .............................................................................................1

CHAPTER 2. LITERATURE REVIEW ...................................................................3

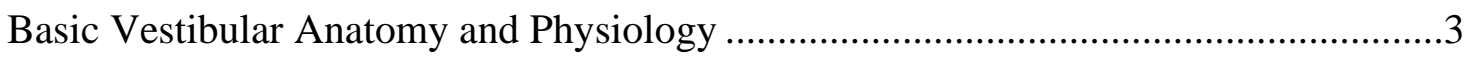

Peripheral Vestibular System..........................................................................

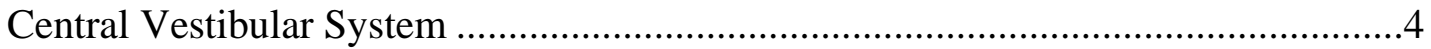

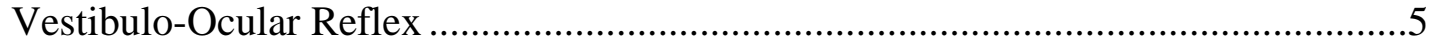

Vestibular Assessment Utilizing the VOR..........................................................6

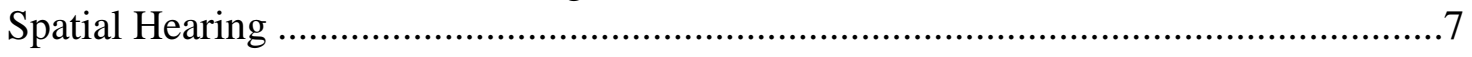

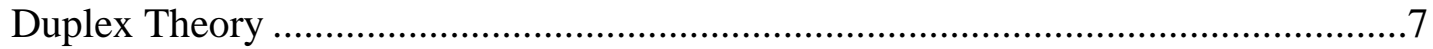

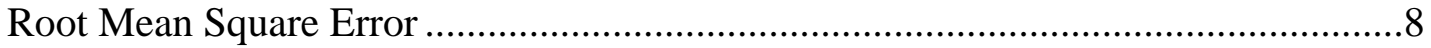

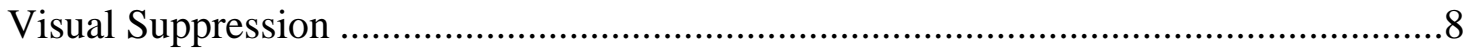

Effects of Non-Visual Stimulation ......................................................................8

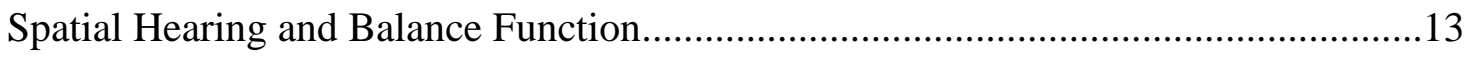

Sound Stimulation and Functional Tests of Balance ............................................13

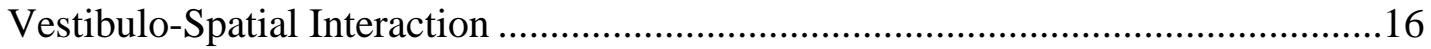

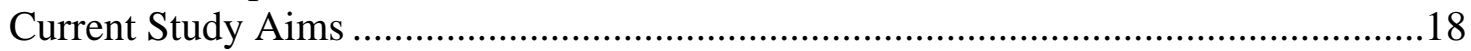

CHAPTER 3. METHODOLOGY .........................................................................20

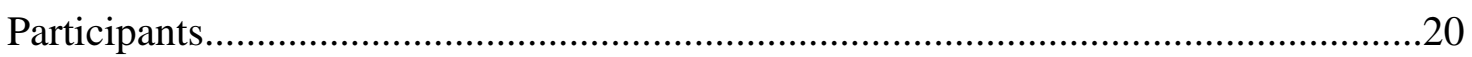

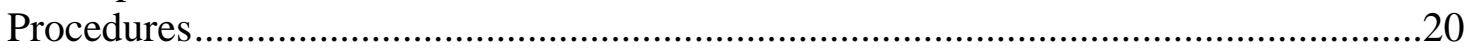

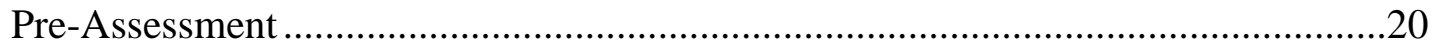

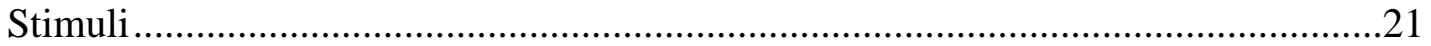

Localization Procedure ....................................................................................21

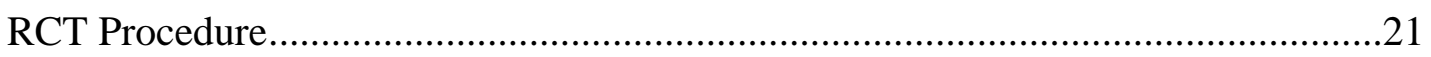

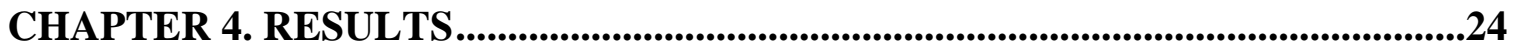

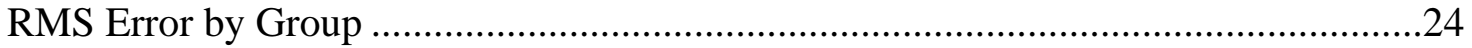

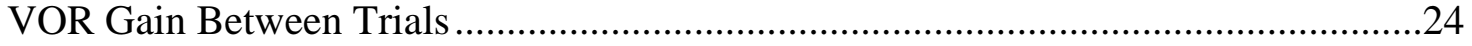

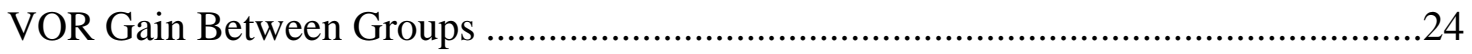

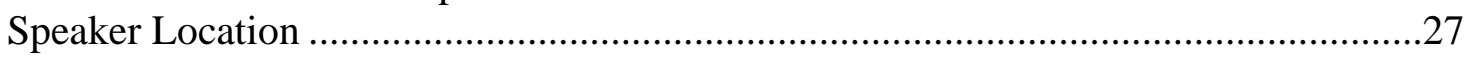

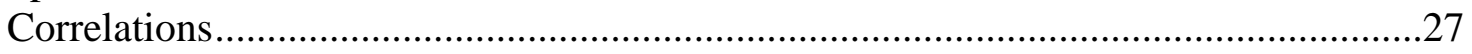

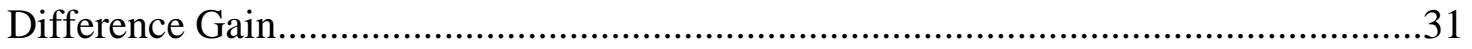

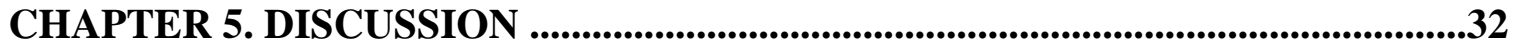

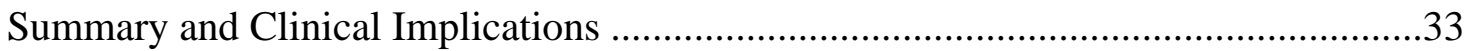

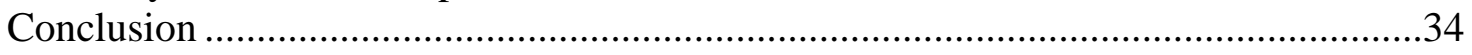

LIST OF REFERENCES .........................................................................................35

VITA.......................................................................................................................................40 


\section{LIST OF TABLES}

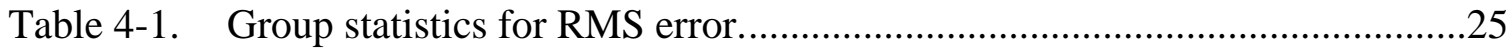

Table 4-2. Independent samples t-test for RMS error between child and adult

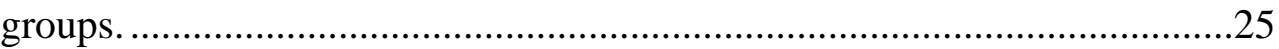

Table 4-3. Paired samples t-test between trials 1 and 2 for child group. ....................25

Table 4-4. Paired samples t-test between trials 1 and 2 for adult group. ....................25

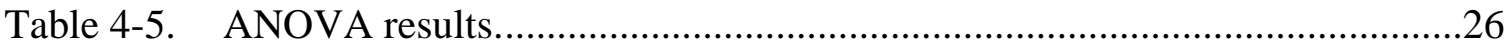

Table 4-6. Significance values of pairwise comparisons for speaker location collapsed across groups.........................................................................28 


\section{LIST OF FIGURES}

Figure 3-1. Localization speaker test set-up......................................................22

Figure 4-1. Mean gain (\%) for each speaker condition by group................................26

Figure 4-2. Linear regression for the child group (moving speaker vs RMS error).......28

Figure 4-3. Linear regression for the child group (fixed speaker vs RMS error)...........29

Figure 4-4. Linear regression for the adult group (moving speaker vs RMS error).......29

Figure 4-5. Linear regression for the adult group (fixed speaker vs RMS error)...........30 


\section{LIST OF ABBREVIATIONS}

\begin{tabular}{|c|c|}
\hline BPPV & Benign Paroxysmal Positional Vertigo \\
\hline CN VIII & Cranial Nerve VIII \\
\hline $\mathrm{COP}$ & Center of Pressure \\
\hline ENG & Electronystagmography \\
\hline ILD & Interaural Level Difference \\
\hline ITD & Interaural Time Difference \\
\hline MOCR & Medial Olivo-cochlear Reflex \\
\hline NIDCD & $\begin{array}{l}\text { National Institute on Deafness and Other Communication } \\
\text { Disorders }\end{array}$ \\
\hline QOL & Quality of Life \\
\hline RCT & Rotational Chair Testing \\
\hline RMS & Root Mean Square \\
\hline SCC & Semicircular Canal \\
\hline SHA & Sinusoidal Harmonic Acceleration \\
\hline SPV & Slow Phase Velocity \\
\hline VOG & Video-oculography \\
\hline VOR & Vestibulo-Ocular Reflex \\
\hline
\end{tabular}




\section{CHAPTER 1. INTRODUCTION}

The ability to maintain postural balance control is essential to daily function and bodily control. This ability relies on proper functioning of the vestibular system. The vestibular system can be considered a "sixth sense" of sort, as it is an automatic, unconscious process that, when impaired, can be a disabling condition. Vestibular disorders are associated with physical symptoms such as lightheadedness, vertigo, nausea, migraines, and general dizziness or instability. Such impairments have a significant quality of life (QOL) and economic impact on individuals and the healthcare system as whole.

Some common vestibular disorders include benign paroxysmal positional vertigo (BPPV), unilateral or bilateral weakness, vestibular migraine, Meniere's disease, and vestibular neuritis. It is estimated $35 \%$ of US adults age 40 years and older have some sort of balance dysfunction, and this incidence rate increases to $85 \%$ for individuals age 80 years and older (Agrawal, Ward, \& Minor, 2013). QOL studies have shown that vertigo from Meniere's disease has an impact similar to other chronic health conditions and is rated between Alzheimer's disease not requiring institutionalization and patients with AIDS or cancer (Anderson \& Harris, 2001; Yardley, Dibb, \& Osborne, 2003).

Ciorba, Bianchini, Scanelli, Pala, Zurlo, \& Aimoni (2017) performed a literature review on QOL indices of general dizziness in the elderly. Using dizziness specific and generic QOL assessment tools the authors found that regardless of underlying pathology (vestibular or non-vestibular) those with chronic or transient dizziness suffer from a significantly deteriorated QOL perception in areas such as isolation, fear, depression, loneliness, and self-esteem. Furthermore, it can lead to significant psychological stress, especially with regards to social isolation and daily function due to increased fear of falling.

The National Institute on Deafness and Other Communication Disorders (NIDCD) reports vestibular disorders are commonly associated with falling, which is the leading cause of injury deaths among adults age 65 or older (Bergen, Stevens, \& Burns, 2016). Moreover, it has also been reported yearly emergency department costs for fallrelated injuries to be approximately $\$ 4$ billion; and projected for fall-related injury and hospitalization rates to increase to $\$ 5.7$ million by the year 2030 (Orces \& Alamgir, 2014).

Kovacs, Wang, \& Grill (2019) attempted to describe the economic impact of vestibular vertigo specifically. The authors reported vestibular vertigo is a contributing factor to rising healthcare costs and one of the reasons this is the case is due to repeated and poor healthcare consultations "on all levels". Moreover, many physicians do not feel competent in differential diagnoses of vertiginous disorders, which can result in increased testing referrals. 
One possible reason for Kovacs et al. (2019) findings of poor consultations and feelings of physician incompetence is it is, unfortunately, the case that there is not a single test which is sensitive and/or specific enough to accurately identify one or all the various vestibular disorders. Therefore, the vestibular assessment protocol is multifactorial and involves the summation of various testing procedures, all of which have varying levels of sensitivity and specificity for accurate disorder identification. For example, the bedside Fukuda Stepping Test has been shown to have up to 50\% sensitivity and $65 \%$ specificity for unilateral vestibular weakness (Honaker, Boismier, Shepard, \& Shepard, 2009); the Dix-Hallpike maneuver has a sensitivity of 50-88\% and specificity up to $75 \%$ for BPPV (Wightman \& Leuty, 1996); the Head Impulse Test has a sensitivity of $35-71 \%$ and specificity of $82-95 \%$ (Wightman \& Leuty, 1996) for unilateral vestibular weakness; and rotational chair (RCT) step velocity testing has a sensitivity of $55 \%$ and a specificity of $84 \%$ for vestibular weakness (Ahmed, Goebel, \& Sinks, 2009).

Due to the QOL and economic impacts it is imperative we have accurate and reliable clinical assessment protocols. It could be postulated that the lower/variable rates of sensitivity and specificity of testing are the result of limited knowledge of the vestibular anatomy and physiology, and how the vestibular system interacts with the other sensory systems. This creates a situation where we are only able to design a test protocol as effective as the depth and breadth of our understanding of the underlying mechanisms. Therefore, it is the aim of this research to aid in improvement of the contemporary vestibular assessment protocol and increase understanding of auditory and vestibular systems interactions. 


\section{CHAPTER 2. LITERATURE REVIEW}

\section{Basic Vestibular Anatomy and Physiology}

The vestibular system is responsible for detecting head position and body movement, and for spatial orientation awareness. All of the physical structures work on the same basic principle of activation of hair cells by an applied external force, such as head/body movement or gravity. This system works in conjunction with the visual and musculoskeletal systems (Shepard \& Telian, 1992) to ensure proper balance function and mobility.

\section{Peripheral Vestibular System}

The peripheral vestibular apparatus is a continuous extension of the inner ear's membranous labyrinth within the petrous portion of the temporal bone. The system consists of three semicircular canals (SCCs), otolithic organs (saccule \& utricle), and the peripheral portion of vestibulocochlear nerve (cranial nerve VIII;(CN VIII)). The system is filled with endolymphatic fluid and contains hair cells. As head/body movement occurs the fluid moves within the system and stimulates the hair cells which are responsible for sending the afferent electrical signals of movement up to the brain. This afferent information is then sent to the efferent visual and musculoskeletal motor systems to aid in balance control. Humans have two sets of each apparatus (left and right), which work in a complementary manner with each other, giving us a 3D awareness of movement and spatial orientation.

The SCCs are responsible for detecting angular or rotary motion. Each SCC is arranged in a specific orientation, making the canals responsible for detection of head movement in the yaw, pitch, and roll planes. While the individual parts of the system work together and might operate to some degree in more than one plane each canal has a primary function of translating acceleration in its respective plane. The hair cells are concentrated at the ends of each canal in a bulbous portion known as an ampulla. As the head moves within its plane of stimulation the endolymph is only displaced in the respective canal, therefore, only the hair cells within that canal ampulla are stimulated upon fluid displacement. The canals lie orthogonally to each other and each canal is one of a coplanar pair with another canal in the opposite ear forming a synergistic pair. The left and right horizontal canals are stimulated simultaneously; the right anterior canal pairs with the left posterior canal; and the left anterior canal pairs with the right posterior canal. The brain receives an opposing signal from each labyrinth (one is inhibited while the other is excited), which gives the system redundancy and causes it to serve as a differential amplifier (Kingma \& Van de Berg, 2016).

Each SCC terminates at the vestibule, which also houses the otolith organs, the utricle and saccule. These organs are situated in the basement membrane of the vestibule with each containing a separate macula (i.e. concentrated hair cell bundle). For the utricle 
the macula is oriented in the horizontal plane (at the opening of the horizontal canal); and for the saccule the macule is located in the sagittal plane on the medial wall (Baloh, Honrubia, \& Kerber, 2010). The maculae are covered by an otoconial membrane into which the hair cells extend. The otoconial membrane is embedded with calcium carbonate crystals called otoconia making it heavier than the surrounding endolymph. During linear movement, the weight of the membrane activates the macular hair cells proportional to the gravitational force and perpendicular to the plane of the other macula (Hamid \& Sismanis, 2006; Baloh, et al., 2010). This unique anatomy of the suspended weighted membrane over the hair cells make them sensitive to gravity and detecting linear motion, or head position relative to gravity (Jamali, Carriot, Chacron, \& Cullen, 2019). Some also argue this gives the otoliths the ability to detect movement in all directions, not just linear movement (Jones, Jones, Mills, \& Gaines, 2009; Miguel, Zarowski, Sluydts, Macias, \& Wuyts, 2020).

The CN VIII has a vestibular and a cochlear (auditory) division. The vestibular division peripherally innervates the vestibular apparatus at multiple sites: the utricle, saccule, and the ampullae of the SCCs. The fibers (aka primary neurons) from these multiple sites converge at the vestibular ganglion prior to merging with the cochlear portion of CN VIII and innervating the brainstem.

\section{Central Vestibular System}

Knowledge of central vestibular pathways is mostly limited to animal studies, with few human studies available (Baloh, et al., 2010). Therefore, we must make general assumptions of anatomy and physiology of the vertebrate and mammal systems and extend it humans (Straka \& Baker, 2013; Matthews, Camp, \& Murray, 2017). The primary neurons originate from the peripheral organs, merge with the cochlear peripheral fibers, and enter the brainstem; thus, beginning the central vestibular system. After encoding afferent signals for a frame of reference for movement and spatial orientation the system is then responsible for aiding in encoding the efferent motor commands to maintain balance and proper spatial navigation (Baloh, et al. 2010; Cullen, 2012; Matthews, et al., 2017).

The primary neurons enter the fourth ventricle of the brainstem extending into secondary order neurons known as vestibular nuclei (VN) (Barmack, 2003; Jones, et al., 2009; Baloh, et al., 2010). There are 4 distinct vestibular nuclei: superior, lateral, medial, and descending. These nuclei act as waystations from which sensory information is received from the periphery then sent to other ascending or descending pathways. The superior VN receives input from the SCCs with some minimal otolith innervation with efferent projections into ocular motor neurons and the cerebellum. The lateral VN receives input from the utricle with efferent projections to the ipsilateral vestibulo-spinal tract and ocular motor neurons. The medial VN receives input from the SCCs, otoliths, contralateral medial $\mathrm{VN}$ with efferent projections to vestibulo-spinal tract, ocular motor neurons, contralateral medial VN, and the cerebellum. The descending VN receives input from the SCCs, otoliths, and the cerebellum with efferent projections to all 4 contralateral 
$\mathrm{VN}$. This area which integrates the $\mathrm{VN}$ as well as sensory and motor afferent and efferent projections collectively is known as the vestibular nuclei complex (Jones, et al., 2009; Baloh, et al., 2010). The efferent projections result in 3 primary vestibular reflexes, the vestibulo-ocular reflex (VOR), vestibulo-colic reflex, and vestibulo spinal reflex (Uchino $\&$ Kushiro, 2011). The purpose of this study is to focus on the VOR alone.

\section{Vestibulo-Ocular Reflex}

The primary goal of the VOR is to stabilize images on the retina to maintain clear and stable vision during head motion. For example, when walking down a street, the ability to focus on a street sign and have it remain clear despite the constant head movement is the direct result of the VOR. The reflex accomplishes this by producing an eye movement that is opposite in direction, but equal in magnitude to the velocity of the head, which causes the eye to remain fixed while the head moves (Jones, et al., 2009; Jacobson \& Shepard, 2008). For example, when the head moves to the right the eyes are translated to the left at the same rate. For a horizontal head movement to the right, the deflection of the cupula in the right horizontal SCC increases the firing rate in the superior vestibular nerve while the deflection of the cupula in left horizontal SCC decreases the firing rate in the left superior vestibular nerve. This asymmetrical change in neural firing is projected through the primary vestibular afferents to the first structures in the central vestibular system referred to as the medial vestibular nuclei (MVN). The MVN are located at the level of the medulla and pontine brainstem and send projections to the oculomotor and abducens nuclei. The oculomotor and abducens nuclei synapse on the effector organs (i.e. extraocular muscle) of the horizontal VOR. In this example of a head turn to the right, the left medial rectus muscle and right lateral rectus will receive an increase in firing and a corresponding contraction that pulls both eyes in a conjugate manner to the left. Conversely, the right medial rectus and left lateral rectus muscles will receive a decrease in the tonic firing rate thereby relaxing allowing the eyes to be pulled to the left. This has the effect of keeping the retinas on the target during the dynamic head movement allowing for clear vision. The eye will eventually reach a point in the orbit where it must be quickly reset to midline. This characteristic eye movement is referred to as nystagmus. Due to the direct innervation of the ocular motor neurons to the vestibular nuclei and its extremely short latency $(7-15 \mathrm{msec})$ this is considered a robust reflex and the easy to measure (Baloh, et al, 2010; Uchino \& Kushiro, 2011; Bronstein, Patel, \& Arshad, 2015).

During motion the nystagmic eye movement works to maintain the eye position relative to the head (Jacobson \& Shepard, 2008; Baloh, et al, 2010). The nystagmus has two phases. First, a slow compensatory phase driven by the vestibular system occurs as the eye drifts away from its center. Second, a fast phase generated by the ocular motor system (paramedian pontine reticular nuclei and MVN) presents as a quick corrective movement, in which the eye returns to center. The direction of nystagmus is qualified by the direction of the fast phase. The relationship between the slow phase velocity (SPV)

and velocity of the head/body movement is known as the VOR gain (VOR gain= slow phase velocity/velocity of head). A perfect correlate (i.e. the SPV and head velocity 
being equal) would be 1 or $100 \%$, as the goal is to create an eye movement that is equal in magnitude, but opposite in direction of the head. However, in clinical measurements gain as low as $15 \%$ can still be considered normal as long as the gain is symmetrical between ears and is assessed using normative values (Jacobson \& Shepard, 2008).

Nystagmus can be measured and VOR gain can be calculated and used to quantify the VOR for differential diagnosis of vestibular dysfunction. As the body moves (i.e. a rotational chair moves) the slow phase of the reflex moves the eye in the opposite direction to compensate for the body movement, whereas the fast phase jerks the eye back to head center when the movement exceeds the orbit of the eye in order be able to continue to reflex in the presence of continuous motion. If you were to take the fast phase out of the tracing of the nystagmus it would result in a sinusoid equal to the sinusoidal rate of the chair movement (which is why the chair rotation is measured in $\mathrm{Hz}$ ). Therefore, when quantifying the VOR we only use the slow phase velocity (SPV) of the reflex and disregard the fast component, as the fast phase component is present only to aid in the functionality of the slow phase component (Baloh, et al., 2010). Recall, the function of the VOR is to create an eye movement that is equal in magnitude, but opposite in direction to the head movement. So, theoretically, a perfect SPV to RCT rate ratio would be 1 or $100 \%$. This ratio between the SPV and RCT rate is known as gain.

Ultimately, the VOR consists of a 3-neuron arc: primary sensory afferent neuron (vestibular nerve), vestibular nucleus neuron, and the oculomotor neuron in the III, IV, or VI brainstem nuclei. When stimulated the vestibular nuclei send excitatory signals to the oculomotor neurons on the opposite side of the brainstem and inhibitory signals to the oculomotor neurons on the same side. Moreover, the flocculo-nodular portion of the cerebellum is known serve as a modulatory control of these multiple projections (Bronstein, Patel, \& Arshad, 2015). Specifically, the flocculus and paraflocculus play a significant role in suppression of the VOR with a visual input and without visual input during vestibular stimulation (Belton \& McCrea, 2000).

\section{Vestibular Assessment Utilizing the VOR}

One of the tests considered the "gold standard" for describing vestibular function via the VOR is the rotational chair test (RCT). This test was first described utilized by Robert Bárány in 1907 to study VOR response decay times following high velocity rotations. (Jacobson \& Shepard, 2008). For this test the participant sits upright in chair with the head tilted downward by approximately 30 degrees thereby placing the h-SCC in the horizontal plane for maximal stimulation. The chair is then oscillated in a side to side motion at various frequencies $(.01-.64 \mathrm{~Hz})$ and the slow component of the nystagmus is measured in relation to the chair movement. This test is referred to as the sinusoidal harmonic acceleration (SHA) test. The rotational chair offers a number of advantages over other tests of semicircular canal function. The test is able to deliver a very controlled stimulus, is highly repeatable, and can test a wide range of frequencies. The primary disadvantage of rotational chair testing is that the laterality of a vestibular impairment is difficult to discern. Testing is performed in either an enclosed chair system in the dark or 
with vision occluded in an open chair system to prevent the central visual fixation mechanisms from reducing/suppressing the measured VOR.

\section{Spatial Hearing}

\section{Duplex Theory}

Spatial hearing (i.e. localization) is the ability to pinpoint the source location of a sound. The dominating theory of azimuthal hearing is the Duplex Theory (Paulus, 2003; Akeroyd \& Whitmer, 2011; and Heijden, Rauschecker, de Gelder, \& Fromisano, 2019). This theory states that there are two disparate binaural cues in mammals, which give essential information to the cortical processing system to interpret from where a sound is originating in the horizontal plane. The first cue is the interaural time difference (ITD) and the second is the interaural level difference (ILD). ITD cues are characterized by the difference in time $(\mu \mathrm{s})$ a sound takes to travel across the head from one ear to the other in the horizontal plane. ILDs are characterized by the difference in perceived level as the sound travels across the head in the horizontal plane. For example, a sound that originates on the left side of the head will reach the left ear sooner (i.e. ITD) and be louder (i.e. ILD) in the left ear than the right. Due to the physical nature of sound waves ITDs are more sensitive to low frequency sounds approximately $<1.5 \mathrm{kHz}$ and ILDs are more sensitive to high frequency sounds $>1.5 \mathrm{kHz}$ (Akeroyd \& Whitmer, 2011; Smith \& Price, 2014). The reason for this frequency specificity of the ITD and the ILDs is the difference in the wavelength. Lower frequencies have longer wavelengths and can travel around the head without the level being disturbed, which results in a timing difference between ears; whereas the shorter wavelengths of higher frequencies are more easily diffracted by the head causing a difference in level between the ears (King, Schnupp, \& Doubell, 2001).

Although it is well studied and clear ITDs and ILDs have a frequency sensitive range, it is also posited as too simplistic a model for sound localization ability measurement. It has also been shown ITDs and ILDs play a role across the frequency spectrum, especially in more complex listening situations such as reverberance or noise (Macpherson \& Middlebrooks, 2002; Heijden, et al., 2019), and therefore do not always adhere to the frequency specific theory. This has been evidenced through animal models which indicate lack of correlation to timing of neural firing to frequency dependent neurons and ITD or ILDs, indicating the cue is still processed despite the frequency with which it was associated (Heijden, et al., 2019). Moreover, ITDs and ILDs are by nature are limited to the horizontal plane localization as sound travels from one side to another and cannot account for the vertical plane or front vs back discrimination (Macpherson \& Middlebrooks, 2002). 


\section{Root Mean Square Error}

While ITDs and ILDs are crucial in localization processing they are not a good functional measurement technique. The root mean square (RMS) error is a method to measure localization ability and is considered the best technique (Yost, Loiselle, Dorman, \& Burns, 2013; Risoud, Hanson, Gauvrit, Renard, Bonne, \& Vincent, 2020), as it measures functional localization performance. RMS error is a standard statistical technique which calculates the standard deviation of the residual values of the linear regression model. In other words, it gives a model of normal distribution of responses in degrees of error for a given response task. This technique has been well established to give us normal means of distribution of error for various age groups with which to compare localization performance (Van Deun, L., Van Wieringen, A., Van Den Bogaert, T., Scherf, F., Offeciers, F. E., Van De Heyning, P. H., ... Wouters, J., 2009; Litovsky \& Godar, 2010; Yost, et al., 2013; Martin, Johnstone, \& Hedrick, 2015).

\section{Visual Suppression}

Depending on the desired task the ocular system may suppress the VOR by overriding the vestibular input, as the VOR is best used when the head is moving, but the object being focused upon is stationary. However, the VOR is suppressed when the head is moving/tracking a moving object, such as a ball being thrown. This occurs because it would be counterintuitive to initiate an eye movement in the opposite direction of the head, as you would lose sight of the ball (Halmagyi and Gresty, 1979). While this suppression is necessary for some instances it is also necessary to prevent visual suppression for accurate measurement when undergoing vestibular assessment. Therefore, vestibular testing is often performed in the dark to prevent an individual from being able to visually fixate on an object and suppress the VOR response.

\section{Effects of Non-Visual Stimulation}

While it is well-established visual input could significantly suppress the VOR the effect of other non-visual input was not known (Barr, Schultheis, \& Robinson, 1976; Moller, White, \& Odvkist, 1990; Jacobson, Piker, Do, McCaslin, \& Hood, 2012; Easterday, Plyler, Lewis, \& Doettl, 2019).

Barr et al. (1976) evaluated the effect of frequency of rotation and mental state in addition to visual stimuli on the VOR. For this study there were a total of 13 participants (age 15-48 years; 12 men, 1 woman). Testing was performed in a rotational chair at rates of $0.3 \mathrm{~Hz}$ and $0.8 \mathrm{~Hz}$ : as well as a "high frequency" (HF) condition using Ganzfeld lenses. Eye movements were recorded using electronystagmography (ENG) and SPV was calculated. Gain was calculated by dividing peak SPV/peak chair velocity for 8-10 half cycles and averaged. Gain was assessed in the following 9 conditions: 
1. Visible stationary target: target stayed on wall while chair oscillated.

2. Imaginary stationary target: participant was instructed to imagine the visual target on the wall.

3. Dark with mental arithmetic: method of arithmetic calculation was not described.

4. Visible moving: target visual target was attached to chair in front of the participant and rotated with the participant.

5. Imaginary moving target: participant was instructed to imagine the moving target from condition 4.

6. Stationary after-image: a cross centered on the fovea was given with a flash gun from the wall.

7. Moving after-image: a cross centered on the fovea was given from the rotating chair.

8. Ganzfeld lens with mental arithmetic in the light.

9. Ganzfeld lens with mental arithmetic in the dark.

Conditions 1-5 were performed at all three frequencies of stimulation $(0.3 \mathrm{~Hz}, 0.8$ $\mathrm{Hz}$, and HF). Conditions 6 and 7 were not performed in the HF condition. Conditions 8 and 9 were performed only at $0.3 \mathrm{~Hz}$. This made for gain being measured in a total of 21 conditions.

While the authors limit their result reporting to descriptive statistics of gain values these values showed a clear trend of gain values changing based on the condition. The authors found large variability in gain ranging $0.32-0.96$ for non-visual conditions, with the moving conditions showing large suppression, the stationary conditions being closest to the desired 1.0, and other conditions falling in the middle of the range. These results led the authors to conclude the VOR is affected by other non-visual factors.

Moller et al. (1990) expanded on the effect of non-visual stimulation on the VOR in a two-part study by utilizing similar conditions to Barr et al. (1976), but also added acoustic and proprioceptive conditions.

Testing was performed in a rotary chair in a darkened booth. Participants were instructed to keep their eyes open and eye movements were recorded using ENG. Gain calculation method was not reported.

Experiment A consisted of 10 participants (age 17-39 years; 4 males, 6 females). The purpose of experiment A was to evaluate gain using a stationary source fixated external to the chair. Gain was assessed in the following 6 test conditions:

1. Darkness with alerting: participant answered questions from a tape-recording during testing.

2. Light stationary: participant was instructed to fixate on a stationary light in center of oscillation.

3. Imaginary stationary light: participant was instructed to imagine the stationary light of the previous condition. 
4. Light + acoustic stationary: participant was instructed to focus on light and stationary click stimulus presented was simultaneously.

5. Acoustic stationary: Same as condition 4 without light.

6. Darkness with alerting: same as first condition.

Each condition was performed at $0.08 \mathrm{~Hz}, 0.16 \mathrm{~Hz}$, and $0.32 \mathrm{~Hz}$ for a total of 18 conditions tested ( 6 conditions $\mathrm{x} 3$ frequencies). Frequency of stimulation and conditions 2-5 were randomly chosen across 3 visits, which were separated by at least 3 days.

Experiment B also consisted of 10 participants (age 18-39 years; 4 males, 6 females). Only 2 participants from experiment A also participated in experiment B. The purpose of experiment B was to evaluate gain using visual, proprioceptive, and acoustic targets. Gain was assessed in the following 7 test conditions:

1. Darkness with alerting: same as experiment A

2. Light moving: Participant was asked to focus on a chair mounted light (i.e. light moved with participant) in front of their eyes during testing.

3. Imaginary light: Participants were instructed to imagine the light from condition 2 during testing.

4. Light proprioceptive moving: the room was lightly dimmed, and participant was instructed to hold their arm outstretched in front of them and observe the thumb throughout testing.

5. Dark proprioceptive moving: Same as condition 4 except the light was turned off and participants were asked to continue to focus on their thumb.

6. Proprioceptive + acoustic moving: Participant was asked to hold the loudspeaker outstretched in front of them during testing

7. Darkness with alerting: same as first condition.

Each of the conditions in experiment B was performed at $0.01 \mathrm{~Hz}, 0.02 \mathrm{~Hz}, 0.04$ $\mathrm{Hz}, 0.08 \mathrm{~Hz}, 0.16 \mathrm{~Hz}$, and $0.32 \mathrm{~Hz}$ with the exception of \#6, which was only performed at $0.08 \mathrm{~Hz}, 0.16 \mathrm{~Hz}$, and $0.32 \mathrm{~Hz}$.

When compared to the baseline condition within each experiment, ultimately, results revealed a significant increase in gain for stationary fixed targets but significant suppression when the acoustic target moved with the participant. In darkness the gain decreased even more significantly with a proprioceptive moving target than during imaginary moving target. These results were consistent with Barr et al. (1976). Furthermore, this study indicated it does not matter whether the stimuli is of visual, acoustic, or imagined in nature, but the perceived location of the target stimuli can affect gain measurements.

More recently, Jacobson et al. (2012) performed an updated two-part study assessing VOR gain in the rotational chair using a VOG system. This is of import note, as the VOG system uses a video recording of the eye rather using the ENG electrode system. This makes the VOG system a more stable measurement technique and the 
current gold standard. The aim of this study was to determine the extent of measurable change in the VOR gain using visual and non-visual stimuli.

Investigation 1 included 16 participants (age 21-30 years; 4 men, 12 women) with normal hearing and reported normal vestibular function. Frequencies tested were $0.02 \mathrm{~Hz}$, $0.08 \mathrm{~Hz}$, and $0.32 \mathrm{~Hz}$ in the rotational chair. The room was set up was dark and the participants were instructed to keep their eyes open. Testing was performed in the following 5 conditions:

1. Mental alerting: Participants were engaged in a mental alerting task (e.g. repeating names).

2. No mental alerting: Participants were simply instructed to keep eyes open.

3. Visual target: Participants were instructed to stare at a chair mounted light, which moved with the participant.

4. Auditory target: Participants were instructed to attend to a chair mounted speaker at $0^{\circ}$, which moved with the participant and emitted a digital recording of classical music at $62 \mathrm{dBA}$.

5. Somatosensory input: Participants were instructed to grasp a bar (mounted to chair) with both hands and stare at it in the dark.

Investigation 2 consisted of 5 participants (age range not reported, mean age $=37$ years; 2 men, 3 women) and aimed to determine if effects of non-visual stimulation were additive. In other words, they questioned if combining auditory and somatosensory input would result in more effect than one or the other alone. They replicated the 5 conditions of investigation 1 and added the following 2 conditions: 1) Imaginary visual target (participants were instructed imagine a light in front of them) and 2) Auditory + somatosensory targets (conditions 4 \& 5 combined). Moreover, for investigation 2 only $0.08 \mathrm{~Hz}$ was utilized.

For each investigation gain was average across trials. Only values for this $0.08 \mathrm{~Hz}$ are reported here for both investigations due to this frequency being the only one used in both investigations within this study.

Generally, results revealed significantly reduced gain for conditions 3-7, but no significant difference between conditions 1 and 2. Of interest, the reduced gain for the auditory condition (\#4) in this study is directly opposed to the increased gain Moller et al. (1990) observed with their investigation A auditory only condition , with Jacobson et al. (2012) showing average gain values of 0.312 and 0.352 and Moller et al. (1990) showing an average gain of 1.01 .

Although limited, previous research has shown that auditory input can influence the VOR with rotational chair testing, however, the underlying mechanism of auditoryvestibular interaction is not understood. Jacobson et al. (2012) postulated two reasons for the observed VOR suppression for the auditory conditions in their study: 1) The neural activation of auditory efferents from the acoustic stimulation spreads to the vestibular 
efferents due to the close proximity of the two systems or 2) the efferent cortical modulation of the VOR was a result of directed attention.

To test the hypotheses proposed by Jacobson et al. (2012) Easterday et al. (2019) measured VOR gain under varying acoustic and attentional conditions in the dark. This study consisted of 17 participants (age 18-34 years; 3 men; 14 women) with normal hearing and vestibular function. They underwent rotational chair testing at $0.02 \mathrm{~Hz}, 0.08$ $\mathrm{Hz}$, and $0.32 \mathrm{~Hz}$. A VOG system was used to measure eye movements. Other measures taken were medial olivo-cochlear reflex (MOCR) thresholds and acoustic reflex thresholds.

To test the first hypothesis the authors measured VOR gain while also playing varying levels of auditory input via insert phones into the participant's ears. The following levels of auditory input were utilized: silent, $42 \mathrm{dBA}, 62 \mathrm{dBA}$, and $82 \mathrm{dBA}$. The silent condition was used as a baseline/control to assess any change with auditory input. Correlational analyses were conducted using VOR gain, the MOCR, and acoustic reflex thresholds, as these are other known tests of auditory efferent activation.

To test the second hypothesis auditory input level conditions were the same, but the acoustic stimuli were time reversed, as such stimuli has been shown to control for non-language attentional functions. Therefore, no instruction to attend to the stimuli was given to the participants. Results revealed gain was not related to acoustic stimulation level, MOCR, the acoustic reflex thresholds, or attentional stimulus type. The authors noted that while group values were not significantly different high inter-participant and intra-participant variability was present, with some individuals showing a large increase in gain with auditory stimulation while showed significant suppression. Post-hoc analysis utilized critical difference scores at an individual level to assess if the variability was still present. While this reduced the amount of observed variability 1 participant still had significant suppression of gain and 3 participants had a significant increase of gain with auditory stimulation.

With respect to the effect of auditory stimulation on VOR gain the results of Easterday et al. (2019), Jacobson et al. (2012), and Moller et al. (1990) were all contrasting, with the studies showing either no difference, suppression, or enhancement, respectively. Easterday et al. (2019) suggested the contrasting differences could be explained by methodological differences of acoustic stimulus delivery. Jacobson et al. (2012) utilized a speaker sound source that moved with the participant, Moller et al. (1990) utilized an externally fixed speaker that did not move with the participant, and Easterday et al. (2019) utilized insert earphones. This led the authors to surmise sound source location within the test environment was the driving force behind differences seen in VOR gain. If the acoustic stimuli moves with the person they will suppress it in an attempt to focus on the stimuli (similar to visually tracking a ball) or if the acoustic stimulation is fixed in space the gain will increase, as the individual searches for the VOR (similar to visually tracking a stationary object while body is in motion). This could cause opposing effects on VOR, as it aims to achieve two different tasks. The removal of these 
spatial cues using the insert earphones in the Easterday et al. (2019) study may have caused the overall gain results to not fully support either of the other studies.

\section{Spatial Hearing and Balance Function}

\section{Sound Stimulation and Functional Tests of Balance}

Data on underlying mechanism/s of spatial hearing and balance function interaction is limited. However, auditory stimulation has been shown to improve postural sway and balance function on several different clinical tests when compared to no sound stimulation using various types of auditory stimuli under various conditions.

Easton, Green, Dizio \& Lackner (1998) studied the effect of auditory cues on bodily sway in 18 sighted or congenitally blind participants (10 sighted, 8 blind; age 2550 years). They were reported as otherwise healthy and physically active, however one blind participant also had a mild hearing loss.

Head and body sway were measured using a center of pressure (COP) technique in which participants stood upright on a force platform with arms at their side. The force platform measured the reaction forces generated by the feet, indicating displacement of the center of pressure. Medial-lateral and anterior-posterior coordinates of foot pressure were computed from the medial-lateral, anterior-posterior, and vertical components. Medial-lateral and anterior-posterior head movements were measured with a video system. The participant was equipped with a headband with an attached LED and the camera tracked the movements of the LED to measure directions of head sway. All signals were sampled at $60 \mathrm{~Hz}$ and collected in real time. There were 4 total conditions: silent, 1 speaker, 2 speakers, and sonar. For the speaker conditions the stimulus was presented through either a single directional speaker positioned directly in front of the participant's head at a distance of $30 \mathrm{~cm}$ or through two speakers, placed $5 \mathrm{~cm}$ adjacent to each left and right ear. Sound stimuli was a $500 \mathrm{~Hz}$ square wave presented at $73 \mathrm{~dB}$. For the sonar condition participants were fitted with a headband equipped with sonar transmitter that emitted a $50-100 \mathrm{~Hz}$ acoustic wave in a $60^{\circ}$ cone in front of the participant, with rationale being individuals could be using echolocation abilities for balance function.

Using the silent condition as a reference results revealed a $10 \%$ decrease in sway on the mean COP in the presence of a $500 \mathrm{~Hz}$ pure tone auditory cue delivered to both sides of the head for both groups with no significant improvement seen for the 1 speaker or sonar conditions.

Zhong \& Yost (2013) studied the effect of auditory cues on the Fukuda Stepping and Tandem Romberg tests in 19 participants (age 18-52 years; 8 men, 11 women) with normal hearing, vision (corrected ok), and balance function. 
In the Fukuda test participants were asked to extend both arms straight in front of their body at $90^{\circ}$ and march in place for 100 steps. Orientation between the midline of their feet was measured pre-test and post-test and angular deviation between the two was calculated for eyes open no sound, eyes closed no sound, and eyes closed with sound conditions. The auditory condition utilized a loudspeaker set up 2 meters in front of the participant which played a broadband noise.

For the Tandem Romberg test participants were instructed to cross their arms and place each hand on its opposite shoulder and hold the stance for 40 seconds. Head sway was measured via an infrared tracker mounted directly above their head in eyes open no sound, eyes closed no sound, and eyes closed with sound conditions. The auditory condition utilized a loudspeaker set up 1 meter in front of the participant which played the broadband noise.

For the Fukuda test angular deviation eyes open vs eyes closed in quiet were compared to each other and eyes closed in quiet vs eyes closed with auditory stimulus were compared to each other. For the Tandem Romberg quiet conditions were compared to each other (eyes open vs closed) and eyes closed conditions (quiet vs noise) were compared to each other. Results indicated a significant improvement for both clinical measures with the addition of the auditory stimulus, with a reported $9 \%$ reduction in sway for the Tandem Romberg and 76\% reduction for the Fukuda Stepping test when compared within the eyes closed conditions.

Siedlecka, Sobera, Sikora, \& Drzewowska (2015) aimed to determine the influence of frequency, spectrum, and loudness of auditory stimulation on posture control in healthy adults. They tested center of pressure amplitude and sway in 29 participants (age not reported; 12 men, 17 women) with normal hearing and balance function. For their study they used binaural stimulation using headphones, as previous research had been performed with loudspeakers as the sound source.

Sway was measured using COP. The participant stood upright on a force platform with feet parallel and hip width apart and arms hanging at their side. COP amplitudes under the feet on the platform were measured medial-lateral and anterior-posterior directions. Sampling rate was $100 \mathrm{~Hz}$ of each COP amplitude; therefore, COP amplitudes was calculated in all directions from the time series.

Participants listened under headphones to stimuli which were altered to be varying, frequencies $(225 \mathrm{~Hz}, 1000 \mathrm{~Hz}$, and $4000 \mathrm{~Hz})$, intensities $(80-120 \mathrm{~dB})$, and spectra (sinusoidal, tonal, broadband noise) played in either a vibrato or crescendo manner. The pure tones were produced by either a guitar or piano, which was previously recorded and played via a $C D$. Each track varied from 10-60 seconds with breaks of 0-60 seconds between tracks. This track time variation was set up such that the participant could not predict the onset time or duration of each track. However, track order was presented with longer tracks at the beginning to reduce fatigue. 
Results revealed only a significant effect for frequency of stimulation with the higher frequency stimulations $(1000 \mathrm{~Hz}$ and $4000 \mathrm{~Hz})$ resulting in a significantly reduced sway amplitude. Moreover, this study addressed male vs female performance of which there was no significant difference. This led the authors to conclude that the higher frequencies play a role in balance function. This was in slight contrast to Easton, et al. (1998) who reported difference for low frequency stimulation only, however, methodological differences (e.g. stimuli type, testing setup, etc.) were present between these studies.

Karim, Rumalla, King, \& Hullar (2017) expanded on Zhong's \& Yost's (2013) work and assessed the effect of spatial landmarks on the Fukuda Stepping test in a twopart experiment. The studied consisted of 8 participants (age $=18-26$ years; 6 men, 2 women) with normal hearing and balance function.

Participants performed the Fukuda test in a total of 7 different conditions. For part 1 of the study the conditions were silence, listening to white noise with headphones, and white noise played from a loudspeaker at $0^{\circ}$. Part 2 of the study then assessed 4 additional conditions of spatial locations at $45^{\circ}, 90^{\circ}, 135^{\circ}$, and $180^{\circ}$ azimuth. For the loudspeaker condition the speaker was positioned $185 \mathrm{~cm}$ from the participant's head at ear level and played at $65 \mathrm{~dB}$ SPL. For the headphone condition participants were instructed to match the volume perceived to the loudspeaker condition. The headphone condition was meant to mimic a head referenced position, whereas the loudspeaker meant to serve as earth-referenced positions. Additionally, participants were blindfolded to prevent the use of visual cues and 3 repetitions of each condition was performed for a total of 21 trials. A goniometer was used to calculate angular deviation (error) in heading direction at the end of each trial.

For part 1 of the study comparisons between the silent, head-referenced, and $0^{\circ}$ earth-referenced conditions were performed. The mean angular deviation was $31.6^{\circ}$ in the silent condition, $28.7^{\circ}$ in the head-referenced condition, and $3.8^{\circ}$ in the $0^{\circ}$ earth-referenced condition. The angular deviations in the silent and head-referenced conditions were not significantly different from each other, but the error in both the silent and headreferenced conditions were significantly worse than the earth-referenced condition.

For part 2 of the study sound source location served as the independent variable. Performance in the earth-referenced condition decreased steady with increasing azimuthal angle from $0^{\circ}$ to $135^{\circ}$ before improving slightly at $180^{\circ}$. The mean error at $45^{\circ}$ angle was $12.3 \mathrm{deg}$, at $90^{\circ}$ angle was $23.4^{\circ}$, at $135^{\circ}$ angle was $29.7^{\circ}$, and at $180^{\circ}$ angle was $21.6^{\circ}$. Performance at 0 and 45 degrees was significantly better than in silence, but not significant for the $90^{\circ}, 135^{\circ}$, or $180^{\circ}$ angles.

These results indicated significant improvement when the sound was earth referenced compared to headphone condition, with a progressive decrease in performance as the azimuth increased from $0^{\circ}$ to $180^{\circ}$. This led the authors to conclude auditory input must be earth-referenced to improve balance function agreeing with previous research that spatial hearing plays a key role observed improvement. 
Results of these studies are in fair agreement with others. Gandemer, Parseihian, Kronland-Martinet, \& Bourdin, (2014) reported a significant improvement in balance function for dynamic auditory stimuli when compared static stimuli (30\% vs $10 \%$, respectively). These results have replicated for white noise stimulation (Ross \& Balasubramaniam, 2015; Zhong \& Yost, 2013), and for other dynamic auditory stimuli (Agaeva \& Altman, 2005; Gandemer, et al., 2014). While there is some debate on the amount of effect or preferred type of stimulus the consensus among researchers is spatial hearing does have a positive effect on posture control and balance function.

\section{Vestibulo-Spatial Interaction}

While it is clear auditory stimulation can have a positive effect on postural stability, and many have concluded it is related to spatial hearing, research is limited to functional assessments of balance and alarmingly few studies have been performed assessing correlations between the VOR and specific spatial cues.

In a 2-part experiment Lewald \& Ehrenstein (1996) assessed the effect of eye position on auditory lateralization (i.e. ILD) on 13 participants (age 19-44 years; 8 men, 5 women) with normal hearing. The aim of this study was to simply examine if adjusting gaze can influence perceived lateralization of the sound as measured by the ILD.

Experiment 1 was performed in a dark and silent room. The head was stabilized and leveled in the azimuthal plan with a chin rest. 5 light diodes in front of the participant were then illuminated to serve as fixation points. Diodes were located at $0^{\circ}$, and $22.5^{\circ}$ and $45^{\circ}$ on both left and right sides. Participants were then fitted with circum-aural headphones through which the ILD acoustic stimulus was played. They were instructed to fix their gaze to the left or right either in the dark or on each light target and maintained fixation while adjusting a sustained continuous stimulus until the stimulus was perceived as being at the center of the head (i.e. ILD measurement).

For the light fixation conditions there were a total of 100 trials (20 trials for each of the five diodes). The mean difference between the ILD adjustments at $-45^{\circ}$ and $+45^{\circ}$ gaze directions was determined at five separate frequencies $(500 \mathrm{~Hz}, 1000 \mathrm{~Hz}, 2000 \mathrm{~Hz}$, $4000 \mathrm{~Hz}$, and $8000 \mathrm{~Hz}$ ). There were 16 trials for each frequency was tested per diode. The SPL of each frequency was corrected such that equal loudness was perceived $(68 \mathrm{~dB}$ at $500 \mathrm{~Hz}, 70 \mathrm{~dB}$ at $1000 \mathrm{~Hz}, 68 \mathrm{~dB}$ at $2000 \mathrm{~Hz}, 65 \mathrm{~dB}$ at $4000 \mathrm{~Hz}, 80 \mathrm{~dB}$ at $8 \mathrm{kHz}$ ). Additionally, measurements were made in the dark (no light diodes). For this portion, participants were instructed to direct their gaze as far as possible to the right or left, and to adjust ILD as described above. 40 trials were made for each left and right gaze directions.

Experiment 2 was a two-alternative forced choice stimulus in which they had to choose whether the ILD stimulus originated from the left or the right while maintaining a fixed gaze on a left or right target. The participant was instructed to fixate on the diode 
and maintain fixation for as long as it was present. Two seconds after target onset, the acoustic target was presented (duration $1 \mathrm{~s}$, rise and fall time $0.1 \mathrm{~s}$ ), after which the diode and acoustic stimulus shut off simultaneously. The participant then had 3 seconds to choose if the sound originated from the left or right. Five seconds after the end of the stimulus, the next trial began. The ILD was varied randomly between trials, over a range from a $4 \mathrm{~dB}$ higher SPL at the left ear to a $4 \mathrm{~dB}$ higher SPL at the right ear, in $1 \mathrm{~dB}$ steps. The position of the fixation targets alternated between $-45^{\circ}$ and $45^{\circ}$. There were a total of 360 trials.

Further measurements followed an identical setup, with the single difference being that the fixation target ended after $1 \mathrm{~s}$, thus providing an interval of $1 \mathrm{~s}$ before the start of the dichotic stimulus. For this portion there was a total 810 trials.

Both experimental parts results indicated a significant psychometric function shift in the direction of the gaze opposite the sound (i.e. sound is localized to the right when gaze is to the left). While this study only assessed purposeful eye gaze not induced by vestibular stimulation it indicated there could be a link between the visual/oculomotor and sound localization systems.

However, Lewald \& Karnath $(2000 ; 2001)$ did evaluate the effect of vestibular stimulation on ILDs. For the first study 13 participants (age 18-49; 6 men, 7 women) with normal hearing were evaluated. The aim of this study was to describe possible vestibular influences on sound localization.

For the ILD task participants were placed in dark and sound-attenuated room with their head fixated by a chin and forehead rest. The participant was instructed to visually fixate on a light diode mounted in front of them at eye level. The acoustic stimulus consisted of continuous dichotic band-pass-filtered noise $(1.5-4 \mathrm{kHz} ; 70 \mathrm{~dB}$ re $20 \mathrm{mPa})$, which was presented via headphones. The participant could vary the ILD by adjusting a potentiometer, thus shifting the sound image toward the left or right. The acoustic stimulus was presented for $12 \mathrm{~s}$. The participant was instructed to adjust the potentiometer such that the sound image was perceived in the median plane of the head. The ILD was recorded automatically. Two seconds after sound offset, the next trial began. Each test condition was comprised of 20 trials and each participant conducted 20 practice trials followed by the three experimental test conditions: no irrigation, irrigation at $37^{\circ}$, and irrigation with ice water. The ILD was also retested 5 minutes after caloric irrigation.

Caloric irrigation was performed to either the right or left ear for 1 min with water at body temperature of $37^{\circ} \mathrm{C}$. During irrigation, the participant sat upright on the chair with the head tilted backward at an angle of $60^{\circ}$. Immediately after irrigation the ILD task was performed. Finally, vestibular stimulation was applied by irrigation in the same ear and manner as in condition 2, but with ice water. Then the induced nystagmus was observed using Frenzel glasses and participants were asked to report if dizziness was felt, and whether it was weak or strong. 
Participants were divided into two separate groups- those with strong dizziness (group 1) and those with weak dizziness (group 2). The authors stated comparisons between no irrigation and irrigation indicated that vestibular stimulation causes a "clear tendency" for ILDs to shift toward the side of stimulation (i.e., the sound level needed amplified at the cooled ear and attenuated at the other ear; meaning the participant's percept was shifted to the non-stimulated ear). However, for follow-up statistical testing they used the $37^{\circ}$ irrigation as the control against the ice water irrigation, which showed a significant shift of the ILD toward the irrigated ear by a mean of $7.3 \mathrm{~dB}$ for group 1 and, which lessened to $2.5 \mathrm{~dB} 5$ minutes after irrigation. It was also reported equivalent shifts were observed in group 2, but with a significantly weaker effect. Group 2 experienced a mean shift of $1.6 \mathrm{~dB}$ between non-irrigated and irrigated ILDs, which remained constant across time trials.

Lewald \& Karnath (2001) then expanded on this previous work with a similarly designed study but evaluated the effect of whole-body rotation (rather than caloric irrigation) on ITDs (rather than ILDs). Results were similar to the previous study, as they found a mean shift of $10.7 \mu$ s to the side of simulation/rotation, with the subjective percept being the sound image shifts towards the non-stimulated ear with vestibular stimulation.

Of important note, Zimmer, Lewald, Erb, Grodd \& Karnath (2004) aimed to study the underlying neural physiology of auditory-visual transformation. They did this by measuring ILDs while participants fixated their gaze to the center, left, or right while simultaneously taking functional magnetic resonance imagining of the neural systems. Results indicated the primary visual cortex activates when the eye is positioned left/right AND with sound lateralization, but not with sound lateralization alone. The authors concluded that while the visual cortex is not directly involved in processing of auditory lateralization it is involved indirectly in the processing of transformation of such auditory cues when eyes are in an eccentric position at the same time auditory input. Neural activation of the visual cortex with auditory stimuli was subsequently supported using event-related potentials (McDonald, Stormer, Martinez, Feng, \& Hillyard, 2013; Feng, Stormer, Martinez, McDonald, \& Hillyard, 2014). Unfortunately, while these studies indicated there is an interaction between the visual and auditory systems no other followup studies or proposed models of the underlying mechanisms of interaction with the vestibular system could be located.

\section{Current Study Aims}

While Lewald \& Karnath $(2000 ; 2001)$ did show some correlation with spatial cues and the VOR, a significant limitation of the current published research in supporting the spatial cues theory is that the body of the research is centered on the effect of sound stimulation on functional tests of balance. Moreover, Jacobson et al. (2012) and Easterday et al. (2019) results lend more toward directed attention playing a role. As such, we still do not have a complete understanding as to why or how auditory spatial cues affect balance function nor any studies looking the relationship of spatial hearing 
and the VOR. Therefore, the present study aims to assess the effect of sound source location and spatial hearing on the VOR. The following research questions were addressed:

1. Does speaker location affect measures of VOR gain?

2. Is RMS error correlated to VOR gain?

Based on previous research it is hypothesized sound source location will significantly affect VOR gain, with a fixed/stationary source resulting in larger gain when compared to a source moving with the participant; and these effects would be larger in an adult group than in a child group.

It is also hypothesized that RMS error will be correlated to sound source location in an adult group, but not in a child group. Specifically, the VOR gain would be highest in participants with better localization and lowest in participants in poorer localization abilities (negatively correlated) for the fixed sound source. However, VOR gain would be positively correlated for the moving sound source and would be lowest in participants with better localization abilities. 


\section{CHAPTER 3. METHODOLOGY}

\section{Participants}

A power analysis indicated 16 participants in each group to be sufficient. Participants were normal hearing individuals with no history of otologic or neurologic dysfunction or disorders who verbally denied taking any drugs/medications known to affect vestibular function. There were two groups of participants: adult age 18-40 years and child age 6-9 years. The rationale of using two such groups is to compare results between a group that has fully developed localization abilities (adults) and a group that has underdeveloped localization abilities (children; Litovsky, 2011). This investigation recruited a total of 23 adults, however, due to the presence of a bilateral weakness in one adult their data was excluded, resulting in data analysis on 22 adults (20 female and 2 male; average age $=23$ years). For the child group a total of 22 ( 9 female and 7 male; average age $=7.5$ years) participants were recruited. However, due to reasons relating to intolerance to the testing and poor equipment fit the data for 6 were excluded, resulting in data analysis on 16 children. For one child participant only one complete run in the silent condition was able to be obtained, however, since there was no significance difference for the group between the two runs the run obtained was utilized twice for data analysis.

This study was approved by the Institutional Review Board at the University of Tennessee, and all participants/guardians signed an informed consent or assent where applicable before participation in this study. All testing was performed at the University of Tennessee Audiology Clinic.

\section{Procedures}

\section{Pre-Assessment}

First, otoscopy was performed to ensure ears were clear of external pathology. Normal middle ear function was assured via tympanometry using the Interacoustics Titan. Normative immittance values are set as follows: $0.9-2.0 \mathrm{~cm} 3$ for ear canal volume, +100 to $-150 \mathrm{daPa}$ for middle ear pressure, and $0.2-2.0 \mathrm{~mL}$ for tympanic membrane peak admittance. Hearing was screened at $20 \mathrm{~dB} \mathrm{HL}$ at 500, 1000, 2000, 4000 and $8000 \mathrm{~Hz}$ in a sound-treated booth using a GSI 61 audiometer with ER-3A insert earphones.

Participants then proceeded to either VOR or spatial hearing measurement. VOR vs. spatial hearing testing order was randomized among participants to account for any possible order effect. 


\section{Stimuli}

The acoustic stimuli set-up for the localization test was the same as utilized in Martin et al. (2015). A Tucker Davis Technologies (TDT) System III (RP2, PM2, AP2) in conjunction with an IBM PC host was used to control stimulus presentation. A custom MATLAB program was used for data collection and stimulus presentation. Stimuli were calibrated prior to every participant using a System 824 Precision Sound Level Meter by Larson Davis.

During testing stimuli were presented at $60 \mathrm{dBA}$ SPL with a $+/-8 \mathrm{~dB}$ rove and consisted of the spondee word "baseball" recorded with a male voice at a sampling rate of $44.1 \mathrm{kHz}$ stored as a .wav file. Roved stimuli were presented randomly on a trial by trial basis to prevent the use of monaural level cues in sound source identification (Martin et al., 2015).

\section{Localization Procedure}

Participants were seated in a sound treated IAC booth $(2.2 . \times 1.8 \mathrm{M})$ at a chairdesk at $0^{\circ}$ azimuth facing a semicircular array of fifteen (15) Cambridge Sound Works Center/Surround IV loudspeakers. Loudspeakers were arranged at $10^{\circ}$ intervals between $-70^{\circ}$ and $70^{\circ}$ azimuth with a radius of $1 \mathrm{~m}$ and matched within $1 \mathrm{~dB} 100-8000 \mathrm{~Hz}$.

Each participant was instructed to continue facing $0^{\circ}$ azimuth toward the center speaker (\#8) and listen for the stimuli (Figure 3-1). Upon hearing the stimulus, they were then allowed to turn their head and click on the computer screen an icon which corresponded to the picture displayed under the speaker from which they believed the stimulus originated. There was not correct/incorrect feedback given regarding responses. However, following each response a puzzle picture appeared piece by piece. Participant was reminded to re-orient to center as needed throughout testing. Unknown to participant only 7 of the 15 speakers were active (every other speaker, even numbered). 3 trials per speaker for a total of 21 trials occurred. Participants were allowed to take breaks as needed.

\section{RCT Procedure}

VOR gain was measured utilizing the Interacoustics NyDiag 200 RCT VOG system. Participants were seated upright in the chair and fit with VOG goggles in a darkened room. Calibration was completed prior to all testing using standard fixed saccade protocol. Each participant underwent SHA testing at a frequency of $0.08 \mathrm{~Hz}$. This SHA frequency was chosen for its stability coupled with decreased time of testing to aid in comfortability/tolerability for children.

There were four (4) test trial conditions: silent, insert phone, moving speaker, and stationary speaker. Each trial was repeated for accuracy, for a total of 16 trials. Order of 


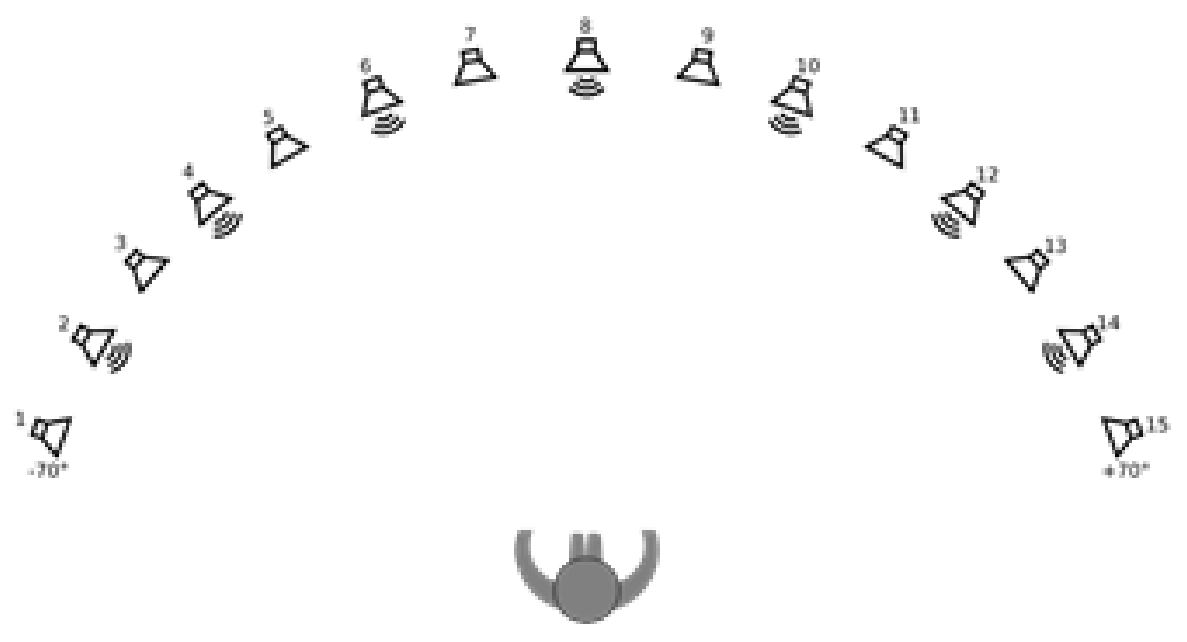

Figure 3-1. Localization speaker test set-up.

Note: Only even numbered speakers were active during testing. 
auditory conditions was randomized. In order to control for possible attention effects participants were told to direct their focus toward the sound source.

For the silent condition, no auditory stimulus was played and the participant was simply instructed to keep eyes open. Foam insert earphones were left in place but turned off to control for extraneous environmental noise. For the insert phone condition, the $3 \mathrm{~A}$ insert earphones coupled to xDuoo media player was utilized. For the moving speaker condition, a speaker mounted to the rotational chair at $0^{\circ}$ azimuth played the stimulus and rotated with the participant, similar to the design of Jacobson et al. (2012). For the stationary speaker condition, the speaker was placed at $+70^{\circ}$ azimuth to the participant's right as a starting point of the (to mimic limit of azimuthal plane of localization study), and remained stationary and unattached to the chair during SHA, similar to the design of Moller et al. (1990). The speaker remained at a $1.5 \mathrm{~m}$ radius (similar to the localization portion of this study) for the stationary speaker target. The stimulus was the same .wav spondee word file as used for the localization portion of this study calibrated to $60 \mathrm{dBA}$ SPL at ear level for each participant prior to testing. The stimulus was played on continuous repeat beginning and ending with chair rotation.

In order to control for possible attention effects participants were asked to focus their attention toward the sound and to keep their eyes open for the auditory conditions. For the silent condition participants were asked to focus on keeping their eyes open and not direct their attention toward any external source. Once a trial was complete the next trial was not commenced until the participant denied experiencing any residual dizziness. Participants were allowed breaks as needed and equipment was recalibrated between breaks. 


\section{CHAPTER 4. RESULTS}

A between subjects repeated measures analysis was utilized for an adult group (18-40 years) vs. The rationale of the two groups was to compare differences in a group with underdeveloped localization abilities (child) and a group with matured localization abilities (adult). A total of 22 children and 23 adults participated in this study. However, one adult participant had to be excluded from analysis due to VOR measuring as having a bilateral vestibular weakness. For the child group a total of 6 participants had to be excluded from data analysis due to reasons such as poor equipment fit, equipment malfunction, or the child's inability to tolerate the testing. Therefore, final statistical analysis was performed with 22 adult participant's data and 16 child participant's data. For one child a single trial (rather than 2) was obtained for the silent VOR condition. Due to analysis indicating repeatability between trials the single data point was used for analysis.

All statistical analysis was performed using IBM SPSS Statistics 26 software.

\section{RMS Error by Group}

An independent samples t-test was performed on the RMS error between the child and adult groups to determine if there was a significant difference in localization ability between groups. Results of the t-test were significant and indicated a mean RMS error of $6.7^{\circ}(\mathrm{SD} \pm 6.04)$ for the child group and $1.6^{\circ}(\mathrm{SD} \pm 1.31$ ) (Tables 4-1 and 4-2).

\section{VOR Gain Between Trials}

Paired samples t-tests were performed to ensure repeatability of VOR gain measures between trials. There were no significant differences between trials for any of the conditions for either group (Tables 4-3 and 4-4); therefore, trials were averaged for each condition for each group (Figure 4-1).

\section{VOR Gain Between Groups}

A two-way repeated measures analyses of variance (ANOVA) was performed to evaluate the effect of speaker location and age group on VOR gain. Speaker location served as the within subjects variable (silent, insert, moving, and fixed) and age group (child, adult) served as the between subjects variable. VOR gain served as the dependent variable. Results indicated a significant main effect for speaker location and for age group; however, the speaker location by age group interaction was not significant (Table 4-5). 
Table 4-1. Group statistics for RMS error.

\begin{tabular}{lccll}
\hline & \multicolumn{4}{c}{ Group Statistics } \\
\cline { 2 - 5 } Group & N & Mean & SD & SE \\
\hline Child & 16 & 6.7 & 6.04 & 1.51 \\
Adult & 22 & 1.6 & 1.31 & 0.28 \\
\hline
\end{tabular}

Table 4-2. Independent samples t-test for RMS error between child and adult groups.

\begin{tabular}{lccccc}
\hline & \multicolumn{5}{c}{ Independent Samples Test } \\
\cline { 2 - 6 } Value & F-Value & Sig. & t & df & Sig (2-tailed) \\
\hline Equal Variances Assumed & 12.013 & 0.001 & 3.840 & 36 & 0.000 \\
Equal Variances Not Assumed & -- & -- & 3.308 & 16.039 & 0.004 \\
\hline
\end{tabular}

Table 4-3. Paired samples t-test between trials 1 and 2 for child group.

\begin{tabular}{lcccccc}
\hline Pair & Mean & SD & SE & t & df & Sig. (2-tailed) \\
\hline Silent 1-2 & 4.5 & 17.04 & 4.26 & 1.06 & 15 & 0.308 \\
Insert 1-2 & 4.06 & 16.74 & 4.19 & 0.97 & 15 & 0.347 \\
Moving 1-2 & 6.44 & 18.29 & 4.57 & 1.41 & 15 & 0.180 \\
Fixed 1-2 & 2.38 & 15.10 & 3.78 & 0.63 & 15 & 0.539 \\
\hline
\end{tabular}

Table 4-4. Paired samples t-test between trials 1 and 2 for adult group.

\begin{tabular}{lcccccc}
\hline Pair & Mean & SD & SE & t & df & Sig. (2-tailed) \\
\hline Silent 1-2 & 0.32 & 10.54 & 2.25 & 0.14 & 21 & 0.89 \\
Insert 1-2 & -0.27 & 10.61 & 2.26 & -0.12 & 21 & 0.91 \\
Moving 1-2 & -1.55 & 14.35 & 3.06 & -0.51 & 21 & 0.62 \\
Fixed 1-2 & 0.05 & 12.42 & 2.65 & 0.02 & 21 & 0.99 \\
\hline
\end{tabular}




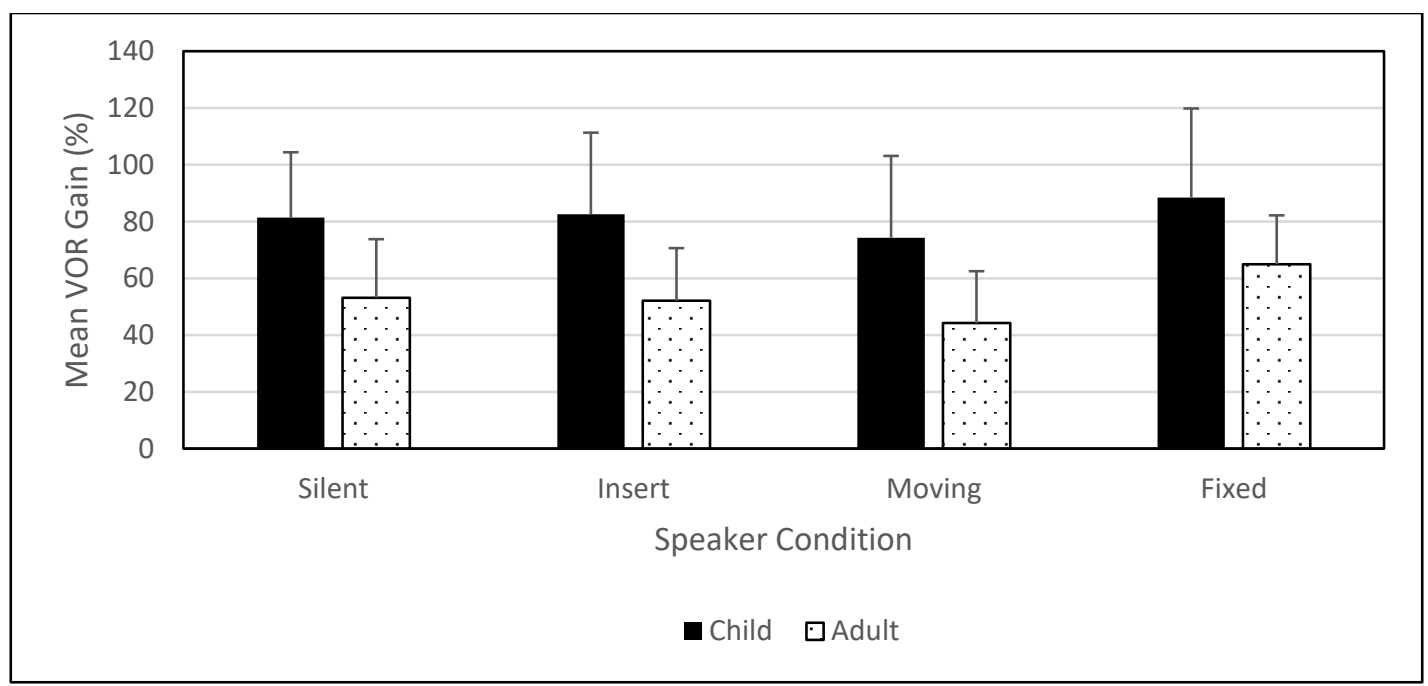

Figure 4-1. Mean gain (\%) for each speaker condition by group.

Table 4-5. ANOVA results.

\begin{tabular}{lcccc}
\hline & \multicolumn{4}{c}{ Multivariate Tests } \\
\cline { 2 - 5 } Condition & F-Value & Significance & Partial Eta Squared & Observed Power \\
\hline Group & 15.87 & 0.000 & 0.91 & 1.00 \\
Speaker & 18.94 & 0.000 & 0.35 & 1.00 \\
Speaker x Group & 0.97 & 0.42 & 0.26 & 0.26 \\
\hline
\end{tabular}




\section{Speaker Location}

Follow-up pairwise comparisons were performed to assess the speaker location main effect (Table 4-6). Results revealed silent and insert conditions not to be significantly different from each other $(\mathrm{p}=1.00)$; however, the moving condition was significantly different from the other 3 conditions and the fixed condition was significantly different from the other 3 conditions. These results indicated children had higher VOR gain than adults. These results further indicated the moving speaker condition significantly reduced VOR gain when compared to the other conditions whereas the fixed speaker condition significantly increased VOR gain when compared to the other conditions.

\section{Correlations}

To answer the second question re: if there was a relationship between localization abilities and VOR gain correlations were run for each group for moving and fixed conditions, as those were the speaker conditions which were statistically significant in the pairwise comparisons.

For the child group, no significant correlation $(\mathrm{p}=0.889$; Figure 4-2) was present for the moving speaker condition and no correlation was seen $(\mathrm{p}=0.810$; Figure 4-3) for the fixed condition.

For the adult group, a significant correlation ( $\mathrm{p}=0.03$; Figure 4-4) was present for the moving speaker condition and for the fixed speaker condition $(p=0.043$; Figure 4-5). 
Table 4-6. Significance values of pairwise comparisons for speaker location collapsed across groups.

\begin{tabular}{llcc}
\hline Speaker 1 & Speaker 2 & Mean Difference & Significance \\
\hline Silent & Insert & -0.075 & 1.00 \\
& Moving & 8.013 & $0.004^{*}$ \\
& Fixed & -9.440 & $0.004^{*}$ \\
Insert & Silent & 0.075 & 1.00 \\
& Moving & 8.088 & $0.012^{*}$ \\
& Fixed & -9.365 & $0.000^{*}$ \\
Moving & Silent & -8.013 & $0.004^{*}$ \\
& Insert & -8.088 & $0.012^{*}$ \\
\multirow{3}{*}{ Fixed } & Fixed & -17.453 & $0.000^{*}$ \\
& Silent & 9.440 & $0.004^{*}$ \\
& Insert & 9.365 & $0.000^{*}$ \\
& Moving & 17.453 & $0.000^{*}$ \\
\hline
\end{tabular}

Note: $*$ indicates significant difference.

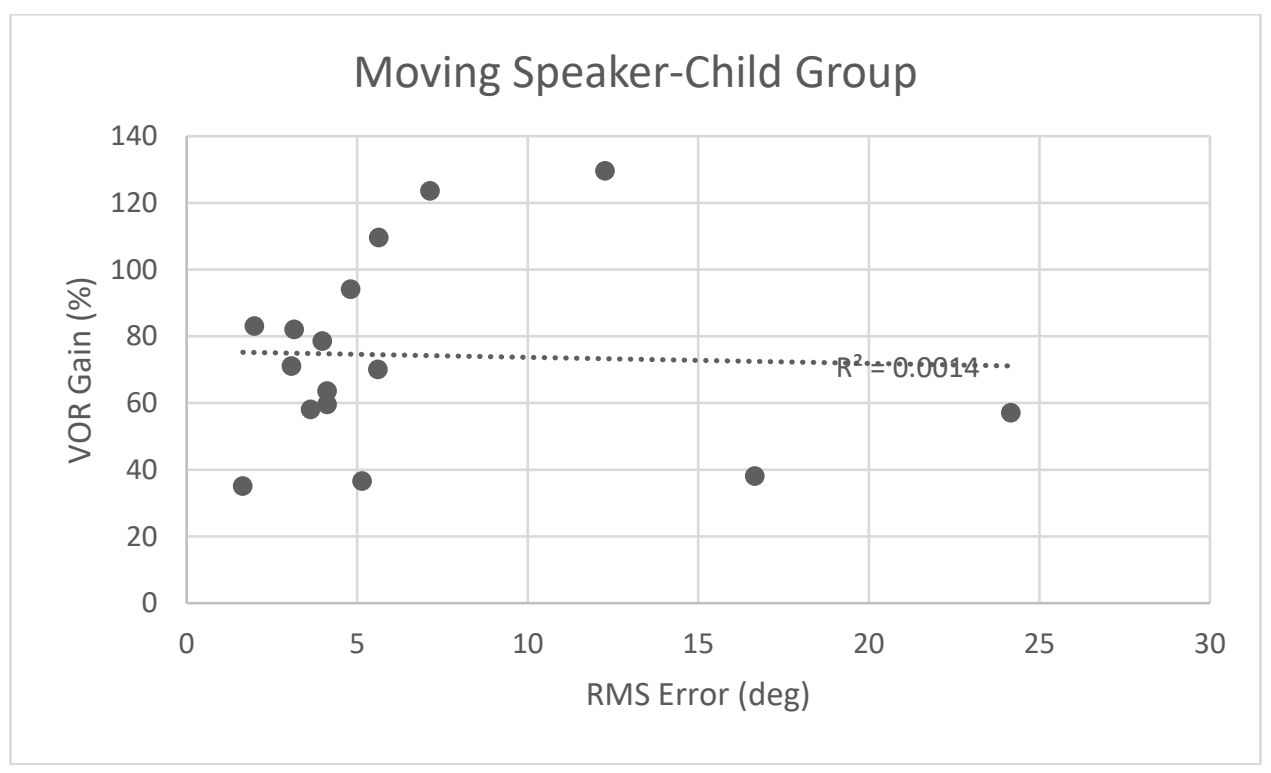

Figure 4-2. Linear regression for the child group (moving speaker vs RMS error). 


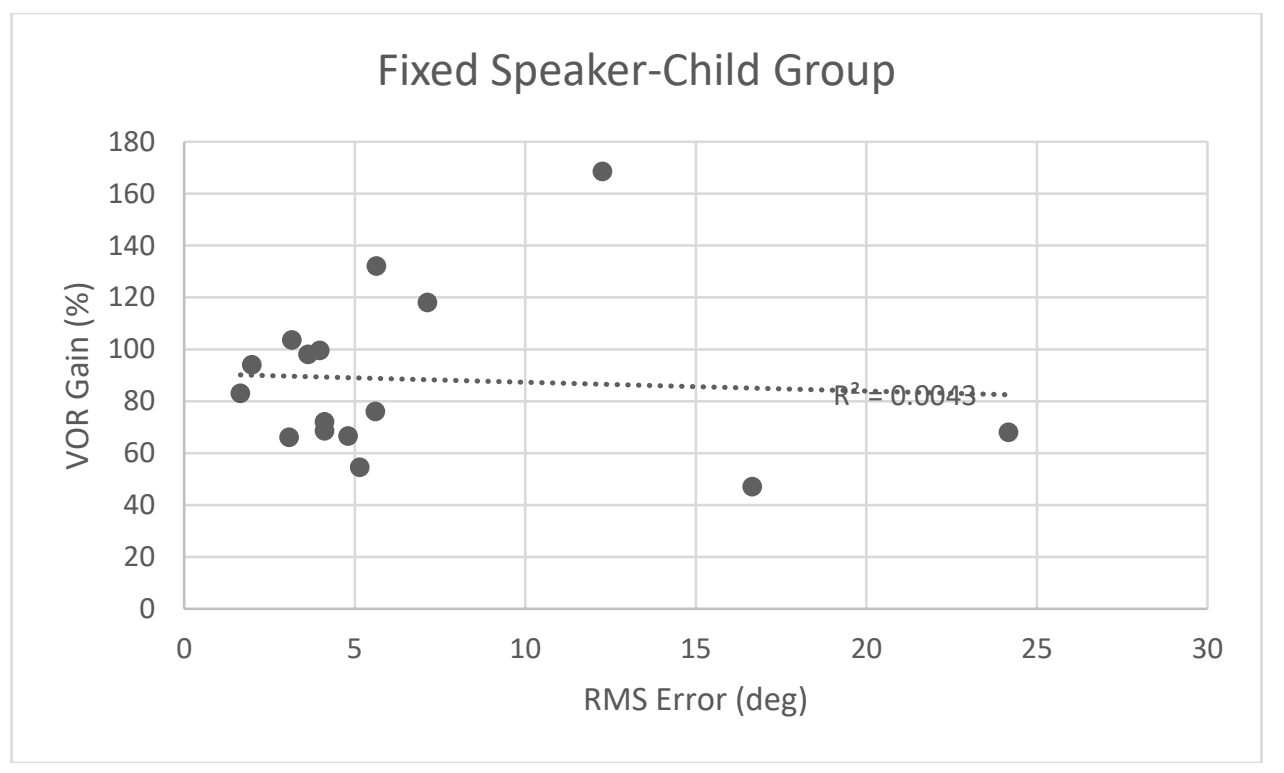

Figure 4-3. Linear regression for the child group (fixed speaker vs RMS error).

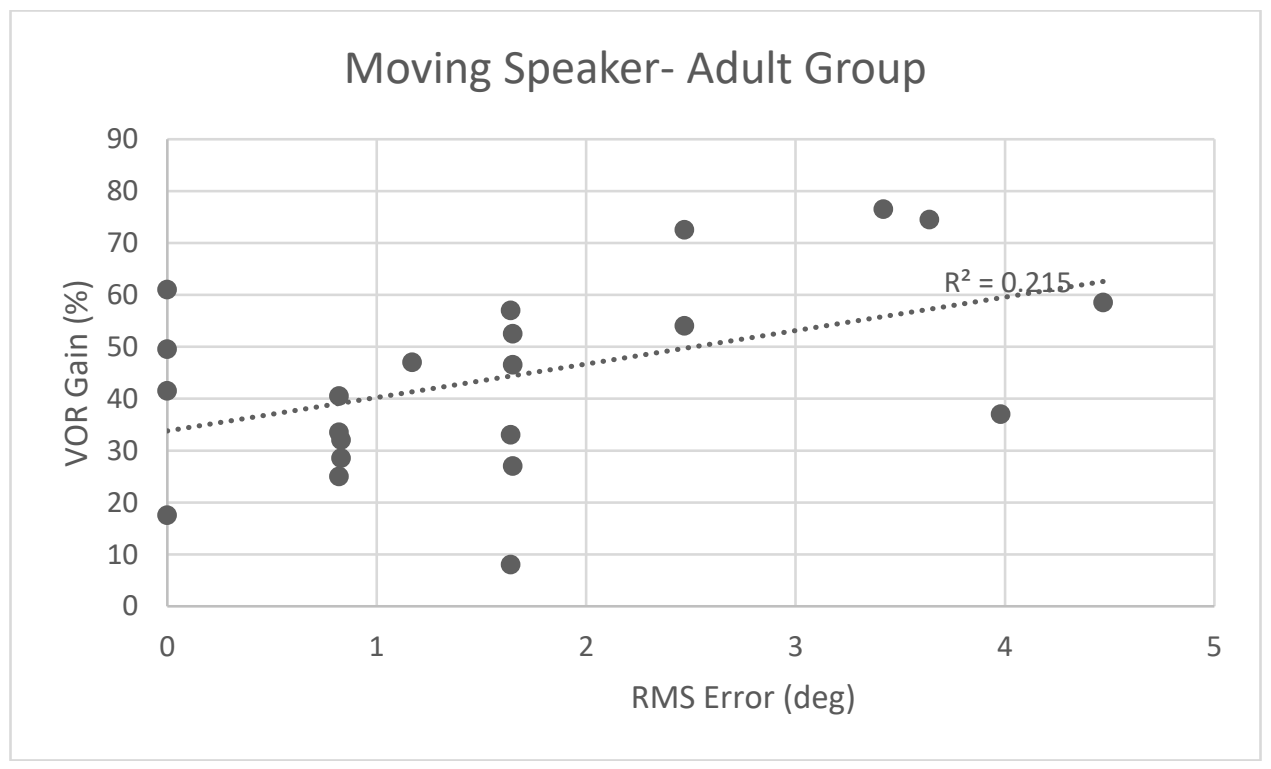

Figure 4-4. Linear regression for the adult group (moving speaker vs RMS error). 


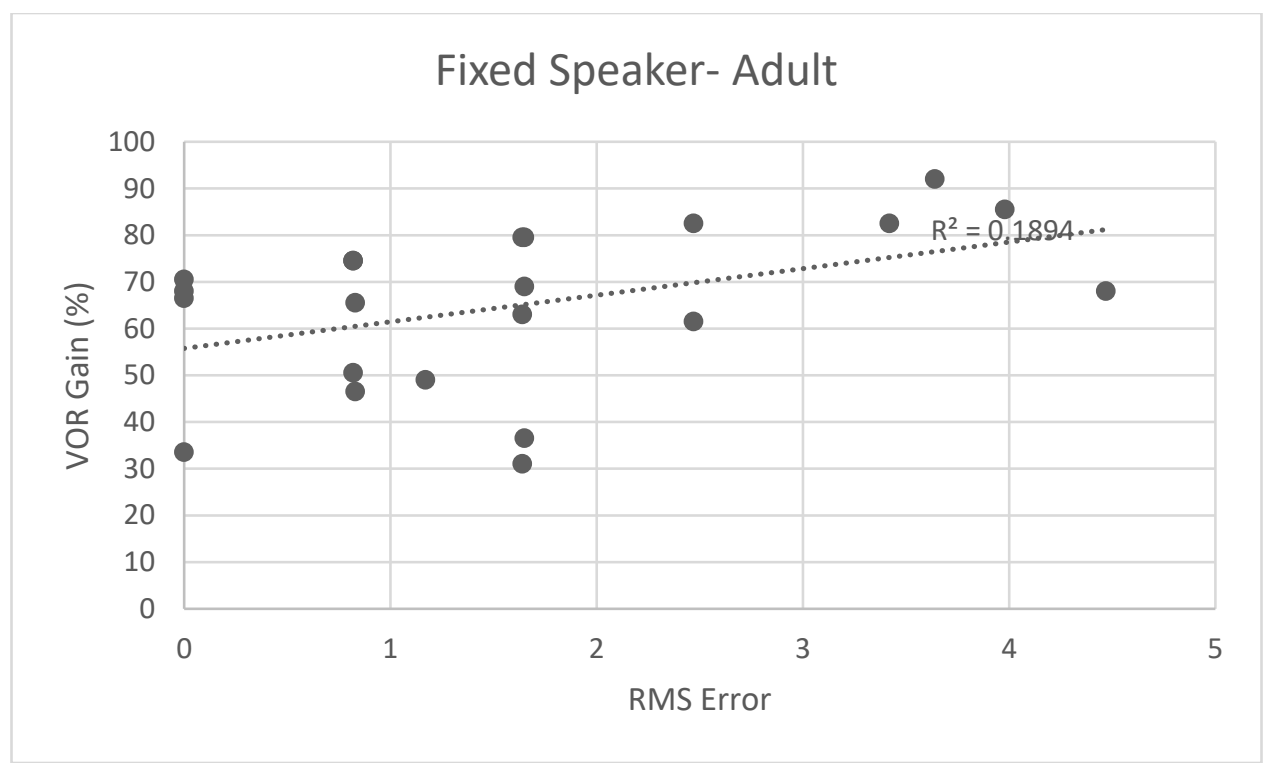

Figure 4-5. Linear regression for the adult group (fixed speaker vs RMS error). 


\section{Difference Gain}

Difference gain was calculated for each group for each speaker location condition. Difference gain was calculated by taking the VOR gain in the auditory condition minus the silent control (baseline) condition (difference gain= VOR gain-baseline gain). This calculation resulted in the amount of change observed, thereby giving us a method to 1) see variability in effect of speaker location on individuals and 2) see if there was a significant change in gain when compared to baseline, with a positive number indicating gain was enhanced and a negative number indicating gain was suppressed.

For the child group, no significant correlation was present for RMSE and measures of suppression $(p=0.922)$ or enhancement $(p=0.896)$. Similarly, for the adult group no significant correlation was present for RMSE and measures of suppression $(\mathrm{p}=$ 0.330 ) or enhancement ( $\mathrm{p}=0.678)$. 


\section{CHAPTER 5. DISCUSSION}

RMSE error was significantly different between the adult and child groups, with the child group having increased error (i.e. poorer localization abilities). These data are consistent with previous research, as Johnstone, Nabelek, \& Robertson (2010) reported a mean RMS error of $7.07^{\circ}$ for the same child age group (compared to $6.7^{\circ}$ for this study) and Martin et al. (2015) reported a mean RMS error of $2.6^{\circ}$ (compared to $1.6^{\circ}$ for this study) for an adult group, both using the same lab and setup (in spite of varying trial numbers). These results indicate good reliability and repeatability of the RMS error task across groups.

There was a significant difference in VOR gain between the child and adult groups, with the children having overall higher gain than the adults across speaker conditions. This is consistent with Chan, Galatioto, Amato, and Kim (2016), who produced RCT normative data stratified by age and reported increased gain for children of similar age groups. Chan, et al. (2016) reported an average gain of $73 \%$ for children of similar age with same SHA frequency whereas the average gain for this study ranged from 74\%-88\% (depending on speaker location). However, Chan et al.'s (2016) study did perform an unspecified alerting task, thereby not controlling for location of any auditory stimulus which could have resulted in their gain to be on the lower limit of our averages.

The first aim of this study was to answer the question if sound source location affected the measurement of the VOR. Results were consistent with the hypothesis and indicated the presence of an external sound in the environment does significantly affect gain while silent and auditory stimulus delivered via insert earphones conditions had similar gain. Moreover, the mere location of the external sound affects if the gain is significantly reduced or enhanced when compared to a silent or insert condition, with a fixed sound source resulting in significantly higher gain and an external sound source moving with the individual resulting in significantly lower gain. This effect was present across child and adult groups.

The second aim of this study was to assess if sound source localization abilities as measured by RMS error and VOR gain was correlated. For the child group there was no correlation. This finding is consistent with the underdeveloped localization system of the group.

However, for the adult group there was a significant positive correlation in both the moving and fixed speaker conditions, which indicates increased RMS error is correlated with increased gain. This finding for the moving condition is to be expected as that condition is meant to suppress gain; therefore, the lower (i.e. better) RMS error would indicative that the individual is better able to locate the sound and "lock in" to the stimulus.

The positive correlation in the fixed condition is an interesting finding, as one instinctively one would expect it to be the opposite and better localization abilities (i.e. 
lower RMS error) to be positively correlated to increased gain in the fixed VOR as the VOR aims to search and lock onto the target. However, this could be indicative of overshoot of the VOR, as one has difficulty locking onto the target once it exceeds a certain angle. This explanation would be consistent with Karim et al.'s (2017) study, who revealed performance on the Fukuda test in the fixed speaker condition decreased steadily with increasing azimuthal angle from $0^{\circ}$ to $135^{\circ}$ before improving slightly at $180^{\circ}$.

Recall for Karim et al.'s (2017) study the mean error for a speaker at located at a $45^{\circ}$ angle was $12.3^{\circ}$ then increased at $90^{\circ}$ to $23.4^{\circ}$ and to $29.7^{\circ}$ at a $135^{\circ}$ angle. Considering the location of the speaker in this study was set to $70^{\circ}$ to mimic the RMS error task this could have inadvertently placed the speaker in the azimuthal range of increased error. This could also explain why the child group has overall increased gain, as the developing VOR system is less efficient in in locking in on a target (such as with the auditory localization system) causing significant overshoot/inflated gain values. This is also evident by the fact a few individual child participants had gain which exceeded $100 \%$.

For the correlation studies performed in the child group, it is also interesting to note that 2 individuals skewed/flattened the regression for both the moving and the fixed conditions and if these two were to be removed the same correlations would be seen as with the adults. This is also consistent with localization data, as this age group is variable and could represent varying degrees of system maturation as the children's system approach adult-like performance.

The lack of significance for the difference gain in both groups indicates that while there is a significant difference in VOR gain between the groups the amount of change remains constant between the two groups.

\section{Summary and Clinical Implications}

Accurate assessment of the VOR is crucial in differential diagnoses of vestibular pathology. Overall, results agree with the theory of previous studies in that individuals utilize spatial hearing when undergoing balance assessment. This is the first study to fully assess the role of spatial placement of auditory cues in the clinical RCT test. This study sheds light on how extraneous auditory cues can affect VOR measurement with RCT assessment. This could aid in easing the aforementioned barriers in the assessment protocol; thus, improving the QOL of an individual and easing the economic burdens placed on the healthcare system.

There are several limitations to this study. The major limitation is the significant need of further studies to corroborate findings. Moreover, it should be noted some report RMS error to be near adult-like by 5 years old; and while we did see significant differences between our groups it could be argued the child age group was nearing a ceiling effect. Additionally, in the interest of time and tolerance for the child group only a single SHA frequency was studied, and the effect on additional frequencies should be assessed. Moreover, the effect of on angle should be further evaluated. Extended age 
groups and disordered populations (such as those with abnormal vestibular function and/or hearing impairment) are also options for future directions of this work.

\section{Conclusion}

The overarching aim of this study was to assess the effect of spatial hearing on the VOR. Results revealed the location of a sound source significantly affect VOR gain for children (age 6-9) years and adults (age 18-40) alike. Moreover, localization abilities as measured by RMS Error are correlated with gain in the adult population when a speaker is fixed to the chair and moves with the participant and when the speaker is fixed externally in the environment.

Ultimately, clinicians should also be aware of the effect of spatial hearing and localization cues within the testing environment and make according adjustments to ensure accurate measurement of the full VOR response; however more research needs to be done to aid in determination of best protocol. 


\section{LIST OF REFERENCES}

Agaeva, MY, Altman, YA. (2005) Effect of a sound stimulus on postural reactions. Hum. Physiol. 31, 511-514. doi: 10.1007/s10747-005-0090-4.

Agrawal Y, Ward BK, Minor LB. (2013) Vestibular dysfunction: Prevalence, impact and need for targeted treatment NIH Public Access. J Vestib Res 23(3):113-117.

Ahmed MF, Goebel JA, Sinks BC. (2009) Caloric Test Versus Rotational Sinusoidal Harmonic Acceleration and Step-Velocity Tests in Patients With and Without Suspected Peripheral Vestibulopathy. Otology \& Neurotology 30(6):800-805.

Akeroyd MA, Whitmer WM. Spatial hearing and hearing aids. ENT Audiol News 20(5):76-79.

Anderson JP, Harris JP. (2001) Impact of Meniere's disease on quality of life. Otol Neurotol 22(6): 888-894.

Baloh RW, Honrubia V, Kerber KA. (2010) Baloh and Honrubia's Clinical Neurophysiology of the Vestibular System. New York: Oxford University Press.

Barmack NH. (2003) Central vestibular system: vestibular nuclei and posterior cerebellum. Brain Res Bull 60(5-6):511-41.

Barr CC, Schultheis LW, Robinson DA. (1976) Voluntary, non-visual control of the human vestibulo-ocular reflex. Acta Otolaryngol 81:365-375.

Belton T, McCrea RA. (2000) Role of the cerebellar flocculus region in cancellation of the VOR during passive whole body rotation. J Neurophysiol 84(3):1599-1613.

doi:10.1152/jn.2000.84.3.1599

Bergen G, Stevens MR, Burns ER. (2016) Falls and Fall Injuries Among Adults Aged $\geq 65$ Years - United States. MMWR Morb Mortal Wkly Rep.

Bronstein AM, Patel M, Arshad Q. (2015). A brief review of the clinical anatomy of the vestibular-ocular connections-how much do we know? Eye 29:163-170.

Chan FM, Galatioto J, Amato M, Kim AH. (2016) Normative data for rotational chair stratified by age. The Laryngoscope 126: 460-463.

Ciorba A, Bianchini C, Scanelli G. et al. (2017) The impact of dizziness on quality-of-life in the elderly. Eur Arch Otorhinolaryngol 274:1245-1250.

Cullen K. (2012) The vestibular system: multimodal integration and encoding of selfmotion for motor control. Trends in Neuroscience 35(3):185-196. 
Easterday MK, Plyer PN, Lewis JD, Doettl SM. (2019) Effect of Auditory Stimulation Level \& Attention on the Vestibulo-Ocular Reflex (VOR). Journal of the American Academy of Audiology 30:792-801.

Easton R, Greene AJ, DiZio P, and Lackner JR. (1998) Auditory cues for orientation and postural control in sighted and congenitally blind people. Exp. Brain Res 118:541-550

Feng W, Störmer VS, Martinez A, McDonald JJ, Hillyard SA. (2014) Sounds activate visual cortex and improve visual discrimination. J Neurosci 34(29):9817-9824. doi:10.1523/JNEUROSCI.4869-13.2014

Gandemer L, Parseihian G, Kronland-Martinet R, and Bourdin C. (2014) The influence of horizontally rotating sound on standing balance. Exp. Brain Res 232:3813-3820

Gandemer L, Parseihian, G, Kronland-Martinet R, and Bourdin C. (2017) Spatial Cues Provided by Sound Improve Postural Stabilization: Evidence of a Spatial Auditory Map. Frontiers in Neuroscience 11:357

Halmagyi GM, Gresty MA. (1979) Clinical signs of visual-vestibular interaction. J Neurol Neurosurg Psychiatry 42:934-939.

Hamid S \& Sismanis A. (2006) Medical Otology and Neurotology: A Clinical Guide to Auditory and Vestibular Disorders. In Medical Otology and Neurotology. Thieme Medical Publishers, Incorporated.

Heijden K, Rauschecker JP, de Gelder B, Formisano E. (2019) Cortical mechanisms of spatial hearing. Nat Rev Neurosci. 20(10):609-623.

Honaker JA, Boismier TE, Shepard NP, \& Shepard NT. (2009) Fukuda Stepping Test: Sensitivity and Specificity. Journal of the American Academy of Audiology 20(5):311314.

Jacobson GP, Piker EG, Do C, McCaslin DL, Hood L. (2012) Suppression of the vestibulo-ocular reflex using visual and nonvisual stimuli. Am J Audiol 21:226-231.

Jacobson G \& Shepard N. (2008) Balance function assessment and management. San Diego, CA: Plural Pub.

Johnstone P, Náblek A, \& Robertson V. (2010) Sound Localization Acuity in Children with Unilateral Hearing Loss Who Wear a Hearing Aid in the Impaired Ear. Journal of the American Academy of Audiology 21(8):522-534.

Jones SM, Jones TA, Mills KN, \& Gaines GC. (2009) Anatomical and Physiological Considerations in Vestibular Dysfunction and Compensation. Seminars in Hearing. 30(4):231-241. 
Karim A, Rumalla K, King L, Hullar T. (2018) The effect of spatial auditory landmarks on ambulation. Gait \& Posture 60:171-174

King AJ, Schnupp JW, Doubell TP. (2001) The shape of ears to come: dynamic coding of auditory space. Trends in Cognitive Science 5(6):261-270.

Kingma H, Van de Berg R. (2016) Anatomy, physiology, and physics of the peripheral vestibular system. Handbook of Clinical Neurology. Editor(s): Joseph M. Furman, Thomas Lempert. 137:1-16

Kovacs E, Wang X, \& Grill E. (2019) Economic burden of vertigo: a systematic review. Health Economics Review 9(1):37.

Lewald J, \& Ehrenstein W. (1996) The effect of eye position on auditory lateralization. Experimental Brain Research 108(3):473-485.

Lewald J, \& Karnath HO. (2000) Vestibular Influence on Human Auditory Space Perception. Journal of Neurophysiology 84(2):1107-1111.

Lewald, J \& Karnath HO. (2001) Sound lateralization during passive whole-body rotation. European Journal of Neuroscience 13(12):2268-2272.

Litovsky RY. (2011) Review of recent work on spatial hearing skills in children with bilateral cochlear implants. Cochlea Implants International 12 Suppl 1(Suppl 1), S30S34.

Litovsky RY \& Godar SP. (2010) Difference in precedence effect between children and adults signifies development of sound localization abilities in complex listening tasks. The Journal of the Acoustical Society of America 128(4):1979-1991.

Macpherson E. \& Middlebrooks J. (2002) Listener weighting of cues for lateral angle: The duplex theory of sound localization revisited. The Journal of the Acoustical Society of America (111): 2219-36.

Martin K, Johnstone P, Hedrick M. (2015) Auditory and visual localization accuracy in young children and adults. International Journal of Pediatric Otorhinolaryngology (79):844-851.

Mathews MA, Camp AJ, Murray AJ. (2017) Reviewing the Role of the Efferent Vestibular System in Motor and Vestibular Circuits. Front Physiol. (8):552.

McDonald JJ, Störmer VS, Martinez A, Feng W, Hillyard SA. (2013) Salient sounds activate human visual cortex automatically. J Neuroscience 33(21):9194-9201. doi:10.1523/JNEUROSCI.5902-12.2013 
Moller C, White V, Odkvist LM. (1990) Plasticity of compensatory eye movements in rotatory tests: II. The effect of voluntary, visual, imaginary, auditory, and proprioceptive mechanisms. Acta Otolaryngol 109:168-178.

Orces CH, Alamgir H. (2014) Trends in fall-related injuries among older adults treated in emergency departments in the USA Injury Prevention. 20:421-423.

Paulus E. (2003) Sound localization cues of binaural hearing. Laryngorhinootologie 82(4):240-8.

Ramos de Miguel A, Zarowski A, Sluydts M, Ramos Macias A, Wuyts FL. (2020) The Superiority of the Otolith System. Audiol Neurootol.25(1-2):35-41. doi: 10.1159/000504595. Epub 2020 Jan 10. PMID: 31927546 ; PMCID: PMC7050676

Ross JM, Balasubramaniam R. (2015) Auditory white noise reduces postural fluctuations even in the absence of vision. Experimental Brain Research 233(8):2357-2363.

Shepard NT, Telian, SA. (1992) System Function. American Journal of Audiology 1(4): 45-51. https://doi.org/10 Balance.1044/1059-0889.0104.45

Siedlecka B, Sobera M, Sikora A, Drzewowska I. (2015). The influence of sounds on posture control. Acta of Bioengineering and Biomechanics 17(3):95-102.

Smith RC, Price SR. (2014) Modelling of human low frequency sound localization acuity demonstrates dominance of spatial variation of interaural time difference and suggests uniform just-noticeable differences in interaural time difference. PLoS One 9(2):e8903

Straka H \& Baker R. (2013) Vestibular blueprint in early vertebrates. Frontiers in neural circuits. (7)182.

Uchino Y, Kushiro K. (2011) Differences between otolith- and semicircular canalactivated neural circuitry in the vestibular system. Neuroscience Research 71(4):315-27.

Van Deun L, Van Wieringen A, Van Den Bogaert, T, Scherf F, Offeciers FE, Van De Heyning, PH ... Wouters J. (2009) Sound localization, sound lateralization, and binaural masking level differences in young children with normal hearing. Ear and Hearing, 30(2): $178-190$.

Wightman M, Leuty L. (1996) Rotational chair testing. In: N.T. Shepard \& S.A. Telian Practical Management of the Balance Disorder Patient. San Diego: Singular Publishing Group, Inc. 109-128.

Yardley L, Dibb B, Osborne G. (2003) Factors associated with quality of life in Meniere's disease. Clinical Otolaryngology and Allied Sciences 28(5):436-441. 
Yost WA, Loiselle L, Dorman M, Burns J, Brown CA. (2013) Sound source localization of filtered noises by listeners with normal hearing: A statistical analysis. Journal of the Acoustical Society of America 133(5):2876-2882

Zhong X, Yost W. (2013). Relationship between postural stability and spatial hearing. Journal of the American Academy of Audiology 24(9):782-788.

Zimmer U, Lewald J, Erb M, Grodd W, Karnath H. (2004). Is there a role of visual cortex in spatial hearing? European Journal of Neuroscience 20:3148-3156. 


\section{VITA}

Mary Easterday was born in Sevierville, TN in 1984. She obtained her undergraduate degree in Speech Language Pathology at the University of Tennessee in Knoxville in 2010. She then went on to obtain her Master's and Doctor of Audiology (Au.D) degrees in 2012 and 2014, respectively, through the University of Tennessee Health Science Center. Upon completion of her Au.D., she began working clinically as an audiologist while concurrently pursing her Ph.D. in Speech and Hearing Science under the direction of Dr. Patrick Plyler. Clinically, she practices predominantly in pediatrics and electrophysiology, whereas her research concentration lies in vestibular assessment. 